\title{
SI: Application of Crabtree/Pfaltz-type iridium complexes for the catalyzed asymmetric hydrogenation of an agrochemical building block
}

\section{General Experimental}

All reactions were performed in oven $\left(130^{\circ} \mathrm{C}\right)$ dried glassware under an atmosphere of dry argon using standard Schleck techniques unless otherwise noted. Chemicals used in this work were purchased from the following vendors:

Reaction solvents: Dichloromethane (anhydrous), THF (anhydrous), isopropanol (anhydrous), 1,4dioxan (anhydrous) were obtained from Acros Organics. 1,2-Dimethoxyethane (anhydrous) was purchased from Sigma Aldrich. 1,1,1,3,3,3-Hexafluoroisopropanol was obtained from Fluorochem and used without further purification.

Reagents: $\quad\left[\operatorname{IrCl}(\mathrm{COD}]_{2} \quad\right.$ and $\quad$ [1,3-Bis(2,6-diisopropylphenyl)imidazol-2-ylidene]chloro[3phenylallyl]palladium(II) were obtained from Strem. Cyclooctadiene, $\mathrm{Pd}\left(\mathrm{PPh}_{3}\right)_{4}$, (dppf) $\mathrm{PdCl}_{2} \cdot \mathrm{CH}_{2} \mathrm{Cl}_{2}$, TBAF (1M THF), ${ }^{n}$ BuLi (1M in hexanes), Chlorodicyclohexylphosphine, Chlorodicyclopentylphosphine, Chlorodiethylphosphine, Chlorodiisopropylphospine were purchased from Sigma Aldrich. Sodium tetrakis[3,5-bis(trifluoromethyl)phenyl]borate was obtained from $\mathrm{ABCR} . \mathrm{K}_{2} \mathrm{CO}_{3}$ and $\mathrm{NaOH}$ were purchased from Fluka, $\mathrm{K}_{3} \mathrm{PO}_{4}$ was obtained from Alfa Aesar. NaOtBu, 9-Phenantrolicbornic acid, 4-(tert-butylphenyl)boronic acid, (2,4,6trimethyl)phenylbornic acid, (4-fluorophenyl)boronic acid, (2,6-dimethylphenyl)boronic acid, (3,5dimethylphenyl)boronic acid, 4-(trifluoromethyl)phenylboronic acid, 4-(methoxy)phenylboronic acid were all obtained from $\mathrm{TCl}$. Catalyst $\mathbf{5 a}$ as well as 2 -chloropyridine building blocks $\mathbf{3 a}$ and $\mathbf{3 b}$ were provided by Bayer AG.

$\mathrm{CDCl}_{3}$ and $\mathrm{CD}_{2} \mathrm{Cl}_{2}$ were purchased from Eurisotop and dried over $3 \AA ̊$ molecular sieves before use.

Column chromatography was performed using Macherey-Nagel, $60 \AA$ silica gel. Visualization was accomplished by UV light and/or potassium permanganate $\left(\mathrm{KMnO}_{4}\right)$, iodine vapor.

Hydrogenation experiments were carried out in $300 \mathrm{~mL}$ autoclave (Parr Instrument Company) in 8-mL glass vials, which were placed inside the autoclave.

${ }^{1} \mathrm{H}$ NMR spectra were recorded using Bruker AV-300 (300 MHz for $\left.{ }^{1} \mathrm{H}\right)$ and Bruker AV-400 (400 MHz for ${ }^{1} \mathrm{H}$ ) spectrometers. ${ }^{13} \mathrm{C}\left\{{ }^{1} \mathrm{H}\right\} \mathrm{NMR}$ spectra were obtained at $75 \mathrm{MHz}$ or $101 \mathrm{MHz} .{ }^{11} \mathrm{~B}\left\{{ }^{1} \mathrm{H}\right\} \mathrm{NMR}$ 


\section{SI: Application of Crabtree/Pfaltz-type iridium complexes for the catalyzed asymmetric hydrogenation of an agrochemical building block}

spectra were obtained at $128 \mathrm{MHz} .{ }^{19} \mathrm{~F}\left\{{ }^{1} \mathrm{H}\right\} \mathrm{NMR}$ spectra were obtained at $282 \mathrm{MHz}$ or $377 \mathrm{MHz}$. ${ }^{31} \mathrm{P}\left\{{ }^{1} \mathrm{H}\right\}$ NMR spectra were obtained at $121 \mathrm{MHz}$ or $162 \mathrm{MHz}$. NMR chemical shifts are reported in parts per million ( $\mathrm{ppm}$ ) downfield from TMS and were referenced to the residual proton resonance and the natural abundance ${ }^{13} \mathrm{C}$ resonance of the solvents. ${ }^{11} \mathrm{~B}$ NMR chemical shifts are reported in parts per million downfield from $\mathrm{BF}_{3} \cdot \mathrm{OEt}_{2}$ and are referenced externally. ${ }^{19} \mathrm{~F}$ NMR chemical shifts are reported in parts per million downfield from $\mathrm{CF}_{3} \mathrm{Cl}$ and are referenced externally. ${ }^{31} \mathrm{P}$ NMR chemical shifts are reported in parts per million downfield from $\mathrm{H}_{3} \mathrm{PO}_{4}$ and are referenced to an external $85 \%$ solution of $\mathrm{H}_{3} \mathrm{PO}_{4}$. Abbreviations used in the reported NMR experiments: $b$, broad; $s$, singlet; $d$, doublet; $t$, triplet; $q$, quartet; $m$, multiplet. All measurements were carried out at room temperature unless otherwise stated.

High resolution mass spectra were recorded on a MAT 95XP ThermoFisher Mass Spectrometer using electrospray ionization mode.

GC Conversion and yields were determined by GC-FID, HP6890 with FID detector, column HP530 $\mathrm{m} \times 250 \mathrm{~mm} \times 0.25 \mu \mathrm{m}$. 
SI: Application of Crabtree/Pfaltz-type iridium complexes for the catalyzed asymmetric hydrogenation of an agrochemical building block

\section{Experimental Procedures}

\subsection{Synthesis Procedures}

2.1.1. Bis(cyclooctadiene)iridium (I) tetrakis(3,5-bis(trifluoromethyl)phenyl)borate

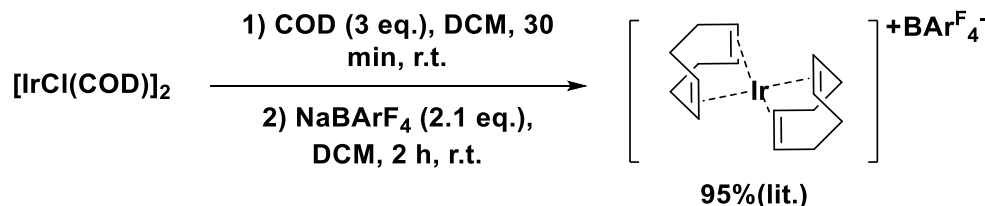

Literature: $\quad$ D. H. Woodmansee, M.-A. Müller, M. Neuburger, A. Pfaltz Chem. Sci., 2010, 1, 72.

Procedure: Chloro(1,5-cyclooctadiene)iridium(I) dimer (130.7 mg, $194.6 \mu \mathrm{mol})$ was added to a dry 25 $\mathrm{mL}$ flask and dissolved by stirring in $8 \mathrm{~mL}$ of absolute DCM. Cyclooctadiene $(63.1 \mathrm{mg}, 71.7 \mu \mathrm{L}, 584 \mu \mathrm{mol})$ was added by syringe and the reaction was stirred for 30 minutes at room temperature. Sodium tetrakis[3,5-bis(trifluoromethyl)phenyl]borate (362 mg, $408.6 \mu \mathrm{mol}$ ) was added as a solid and the very dark red solution was stirred for 2 hours at room temperature. The solution was filtered through a 2 gram silica pipette column which was washed with an additional $7 \mathrm{~mL}$ of DCM. The solvent was concentrated at a rotovap to dryness. The resulting crude mixture was taken up in $5 \mathrm{~mL}$ of DCM, filtered through a micron filter and layered with $5 \mathrm{ml}$ of hexane. Crystallization at $-20^{\circ} \mathrm{C}$ overnight followed by cold vacuum filtration and washing with $-20^{\circ} \mathrm{C}$ hexane $(2 \times 25 \mathrm{~mL})$ provided very large dark red high symmetry crystals of X-ray diffraction quality which were dried on a high vacuum for 3 hours to provide $470 \mathrm{mg}$ of pure product $(370 \mu \mathrm{mol}, 95 \%)$.

Analytical data (found): ${ }^{1} \mathrm{H}-\mathrm{NMR}\left(300 \mathrm{MHz}, \mathrm{CD}_{2} \mathrm{Cl}_{2}\right) \delta(\mathrm{ppm})=7.83-7.64(\mathrm{~m}, 2 \mathrm{H}), 7.56(\mathrm{~s}, 1 \mathrm{H}), 5.29-$ $4.92(\mathrm{~m}, 2 \mathrm{H}), 2.59-2.22(\mathrm{~m}, 4 \mathrm{H}) .{ }^{19} \mathrm{~F}$ NMR $\left(282 \mathrm{MHz}, \mathrm{CD}_{2} \mathrm{Cl}_{2}\right) \delta(\mathrm{ppm})=-63.1$.

\subsubsection{General procedure for the Suzuki cross couplings}
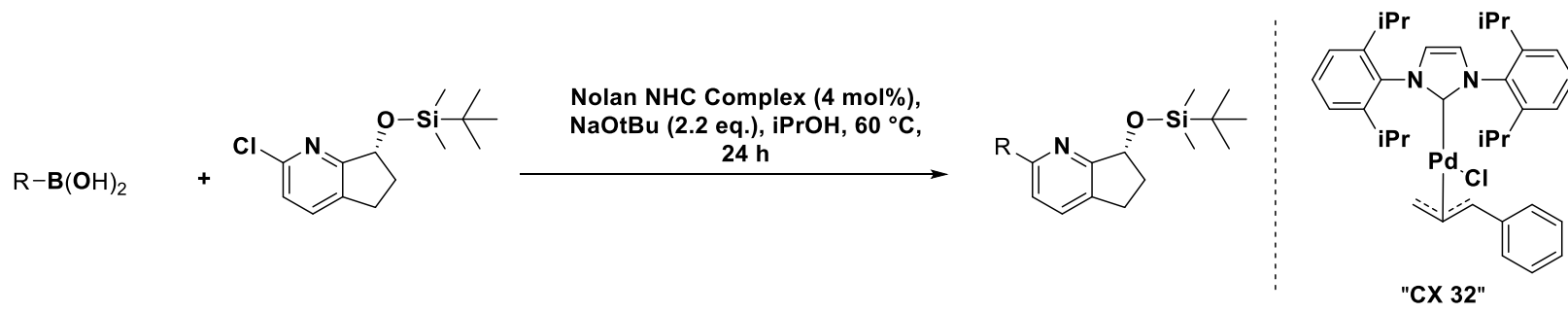

Literature: $\quad$ According to the synthesis route described in D. H. Woodmansee, M.-A. Müller, M. Neuburger, A. Pfaltz Chem. Sci., 2010, 1, 72.

Reaction (under argon): (S)-7-(tert-butyldimethylsilyloxy)-2-chloro-6,7-dihydro-5 $\mathrm{H}$ cyclopenta[b]pyridine (3a, $1 \mathrm{mmol}$ ), boronic acid ( $2 \mathrm{mmol}, 2$ eq.), CX 32 (40 $\mu \mathrm{mol} ; 4 \mathrm{~mol} \%$ ) and freshly 


\section{SI: Application of Crabtree/Pfaltz-type iridium complexes for the catalyzed asymmetric hydrogenation of an agrochemical building block}

degassed $\mathrm{PrOH}(15 \mathrm{~mL}$ ) were added to a $50 \mathrm{~mL}$ Schlenk tube containing a PTFE coated stirring bar, followed by a solution of $\mathrm{NaOtBu}(2.2 \mathrm{mmol}, 2.2 \mathrm{eq})$ in $\mathrm{iPrOH}$. The reaction was stirred at $60^{\circ} \mathrm{C}$ for 24 hours.

Work-up (under air): The reaction was worked up by extraction with DCM $(25 \mathrm{~mL})$ and water $(2 \times 25$ $\mathrm{mL})$, followed by brine $(1 \times 25 \mathrm{~mL})$. The organic layer was dried over magnesium sulfate, filtered and concentrated at the rotary evaporator to provide crude product which was purified by column chromatography on a flash column with a heptane/DCM gradient starting from pure heptane changing gradually to pure DCM.

\subsubsection{1. (R)-7-((tert-butyldimethylsilyl)oxy)-2-phenyl-6,7-dihydro-5H-} cyclopenta $[b]$ pyridine

The reaction was performed twice in our laboratory according to the

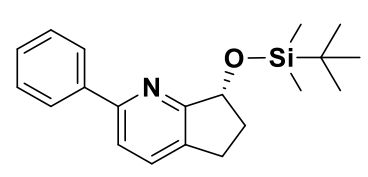
above described procedure (1.1.2.). In the first run (1 mmol scale) the product could be isolated as a white solid in $86 \%$ yield. In the second run (6.9 mmol) a catalyst loading of $6 \mathrm{~mol} \%$ was used. This run gave a slightly lower yield of $85 \%$.

${ }^{1} \mathrm{H}-\mathrm{NMR}\left(300 \mathrm{MHz}, \mathrm{CDCl}_{3}\right): \delta(\mathrm{ppm})=8.14-8.02(\mathrm{~m}, 2 \mathrm{H}), 7.66-7.53(\mathrm{~m}, 2 \mathrm{H}), 7.52-7.34(\mathrm{~m}, 3 \mathrm{H})$, $5.33-5.21(\mathrm{~m}, 1 \mathrm{H}), 3.15-2.97(\mathrm{~m}, 1 \mathrm{H}), 2.87-2.71(\mathrm{~m}, 1 \mathrm{H}), 2.58-2.39(\mathrm{~m}, 1 \mathrm{H}), 2.16-1.97(\mathrm{~m}$, 1H), 1.00 (s, 9H), $0.30(\mathrm{~s}, 3 \mathrm{H}), 0.23(\mathrm{~s}, 3 \mathrm{H}) .{ }^{13} \mathrm{C}-\mathrm{NMR}\left(75 \mathrm{MHz}, \mathrm{CDCl}_{3}\right) \delta(\mathrm{ppm})=164.7,156.1,139.8$, $134.8,133.5,128.7,128.6,126.9,119.3,76.3,34.5,27.3,26.1,18.8,-4.0,-4.5$.

2.1.2.2. (R)-7-((tert-butyldimethylsilyl)oxy)-2-(4-methoxyphenyl)-6,7-dihydro-5Hcyclopenta[b]pyridine

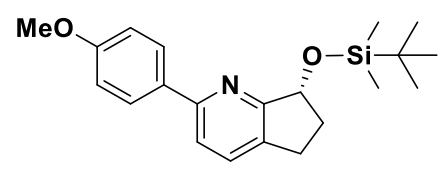

The reaction was performed once in our laboratory $(3.3 \mathrm{mmol}$ scale) according to the above described procedure (1.1.2.). The product could be isolated as a white solid with a yield of $84 \%$.

${ }^{1} \mathrm{H}-\mathrm{NMR}\left(300 \mathrm{MHz}, \mathrm{CDCl}_{3}\right): \delta(\mathrm{ppm})=8.09-7.94(\mathrm{~m}, 2 \mathrm{H}), 7.53(\mathrm{~s}, 2 \mathrm{H}), 7.05-6.92(\mathrm{~m}, 2 \mathrm{H}), 5.24(\mathrm{t}$, $J=6.1 \mathrm{~Hz}, 1 \mathrm{H}), 3.86(\mathrm{~s}, 3 \mathrm{H}), 3.13-2.95(\mathrm{~m}, 1 \mathrm{H}), 2.84-2.68(\mathrm{~m}, 1 \mathrm{H}), 2.55-2.35(\mathrm{~m}, 1 \mathrm{H}), 0.98(\mathrm{~s}$, 9H), $0.28(\mathrm{~s}, 3 \mathrm{H}), 0.21$ (s, 3H). ${ }^{13} \mathrm{C}-\mathrm{NMR}\left(75 \mathrm{MHz}, \mathrm{CDCl}_{3}\right) \delta(\mathrm{ppm})=164.5,160.2,155.8,134.0,133.4$, $132.5,128.1,118.6,114.1,76.3,55.5,34.5,27.3,26.1,18.8,-4.0,-4.5$. 
SI: Application of Crabtree/Pfaltz-type iridium complexes for the catalyzed asymmetric hydrogenation of an agrochemical building block

2.1.2.3. (R)-2-(anthracen-9-yl)-7-((tert-butyldimethylsilyl)oxy)-6,7-dihydro-5Hcyclopenta $[b]$ pyridine

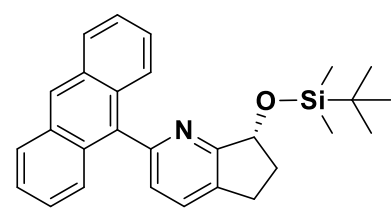

The reaction was performed once in our laboratory $(2.2 \mathrm{mmol}$ scale) according to the above described procedure (1.1.2.). The product could be isolated as orange solid with a yield of $43 \%$.

${ }^{1} \mathrm{H}-\mathrm{NMR}\left(300 \mathrm{MHz}, \mathrm{CDCl}_{3}\right): \delta(\mathrm{ppm})=8.52(\mathrm{~s}, 1 \mathrm{H}), 8.09-7.99(\mathrm{~m}, 2 \mathrm{H}), 7.84-7.64(\mathrm{~m}, 3 \mathrm{H}), 7.50-$ $7.40(\mathrm{~m}, 2 \mathrm{H}), 7.38-7.30(\mathrm{~m}, 3 \mathrm{H}), 5.34-5.22(\mathrm{~m}, 1 \mathrm{H}), 3.35-3.20(\mathrm{~m}, 1 \mathrm{H}), 3.02-2.89(\mathrm{~m}, 1 \mathrm{H}), 2.58$ $-2.42(\mathrm{~m}, 1 \mathrm{H}), 2.26-2.12(\mathrm{~m}, 1 \mathrm{H}), 0.90(\mathrm{~s}, 9 \mathrm{H}), 0.15(\mathrm{~s}, 3 \mathrm{H}), 0.08(\mathrm{~s}, 3 \mathrm{H}) .{ }^{13} \mathrm{C}-\mathrm{NMR}\left(75 \mathrm{MHz}, \mathrm{CDCl}_{3}\right)$ $\delta(\mathrm{ppm})=165.1$, 156.3, 135.2, 132.9, 132.5, 131.5, 131.4, 130.3, 130.1, 128.5, 128.2, 127.3, 127.0, $126.3,126.1,125.5,125.1,124.9,76.2,34.3,27.9,25.9,18.4,-4.1,-4.6$.

2.1.2.4. (R)-2-(4-(tert-butyl)phenyl)-7-((tert-butyldimethylsilyl)oxy)-6,7-dihydro$5 H$-cyclopenta $[b]$ pyridine

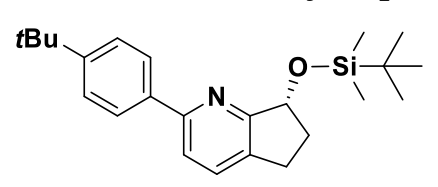

The reaction was performed once in our laboratory ( $3 \mathrm{mmol}$ scale) according to the above described procedure (4.1.5.). The product could be isolated as white solid with a yield of $80 \%$.

${ }^{1} \mathrm{H}-\mathrm{NMR}\left(300 \mathrm{MHz}, \mathrm{CDCl}_{3}\right): \delta(\mathrm{ppm})=8.10-8.01(\mathrm{~m}, 2 \mathrm{H}), 7.62-7.48(\mathrm{~m}, 4 \mathrm{H}), 5.34-5.21(\mathrm{~m}, 1 \mathrm{H})$, $3.13-3.02(\mathrm{~m}, 1 \mathrm{H}), 2.86-2.74(\mathrm{~m}, 1 \mathrm{H}), 2.55-2.43(\mathrm{~m}, 1 \mathrm{H}), 2.16-2.03(\mathrm{~m}, 1 \mathrm{H}), 1.04(\mathrm{~s}, 9 \mathrm{H}), 0.34$ $(\mathrm{s}, 3 \mathrm{H}), 0.27(\mathrm{~s}, 3 \mathrm{H}) .{ }^{13} \mathrm{C}-\mathrm{NMR}\left(75 \mathrm{MHz}, \mathrm{CDCl}_{3}\right) \delta(\mathrm{ppm})=164.6,156.1,151.6,137.0,134.4,133.3$, $126.6,125.6,119.0,76.3,34.8,34.5,31.5,27.3,26.2,18.8,-4.0,-4.5$.

2.1.2.5. (S)-7-((tert-butyldimethylsilyl)oxy)-2-(4-fluorophenyl)-6,7-dihydro-5Hcyclopenta[b]pyridine

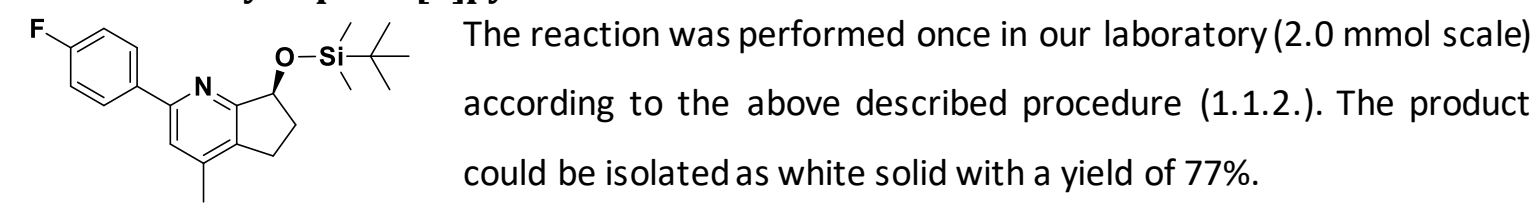

${ }^{1} \mathrm{H}-\mathrm{NMR}\left(300 \mathrm{MHz}, \mathrm{CDCl}_{3}\right): \delta(\mathrm{ppm})=8.10-7.90(\mathrm{~m}, 2 \mathrm{H}), 7.41-7.29(\mathrm{~m}, 1 \mathrm{H}), 7.12(\mathrm{dd}, \mathrm{J}=9.0,8.5$ $\mathrm{Hz}, 2 \mathrm{H}$ ), 5.23 (dd, J = 7.1, $5.0 \mathrm{~Hz}, 1 \mathrm{H}$ ), 2.99 (ddd, J = 16.2, 8.8, 4.8 Hz, 1H), 2.71 (ddd, J = 16.2, 8.4, $6.0 \mathrm{~Hz}, 1 \mathrm{H}$ ), 2.46 (dddd, J = 13.3, 8.4, 7.1, $4.9 \mathrm{~Hz}, 1 \mathrm{H}$ ), 2.31 (d, $\mathrm{J}=0.8 \mathrm{~Hz}, 3 \mathrm{H}$ ), 2.05 (dddd, $\mathrm{J}=13.2$, 8.8, 6.0, $5.0 \mathrm{~Hz}, 1 \mathrm{H}), 0.98(\mathrm{~s}, 9 \mathrm{H}), 0.27(\mathrm{~s}, 3 \mathrm{H}), 0.20(\mathrm{~s}, 3 \mathrm{H}) .{ }^{13} \mathrm{C}-\mathrm{NMR}\left(75 \mathrm{MHz}, \mathrm{CDCl}_{3}\right) \delta(\mathrm{ppm})=$ $164.8,162.8\left(\mathrm{~d},{ }^{1} \mathrm{~J}=187 \mathrm{~Hz}\right), 155.2,144.1,136.0,134.1,128.5\left(\mathrm{~d},{ }^{3} \mathrm{~J}=8.3 \mathrm{~Hz}\right), 120.0,115.4\left(\mathrm{~d},{ }^{2} \mathrm{~J}=\right.$ $21.4 \mathrm{~Hz}), 76.3,33.8,26.0,25.8,18.8,18.6,-4.2,-4.6 .{ }^{19} \mathrm{~F}-\mathrm{NMR}\left(282 \mathrm{MHz}, \mathrm{CDCl}_{3}\right) \delta(\mathrm{ppm})=-114.3$. 
SI: Application of Crabtree/Pfaltz-type iridium complexes for the catalyzed asymmetric hydrogenation of an agrochemical building block

2.1.2.6. (S)-7-((tert-butyldimethylsilyl)oxy)-4-methyl-2-phenyl-6,7-dihydro-5Hcyclopenta[b]pyridine

The reaction was performed once in our laboratory (3.0 mmol scale),<smiles>Cc1cc(-c2ccccc2)nc2c1CCC2O[Si](C)(C)C(C)(C)C</smiles>
using boronic acid (7.5 mmol, 2.5 eq.), Nolan NHC catalyst (4 mol\%) and NaOtBu (2.5 eq) according to the above described procedure (1.1.2.). The product could be isolated as white solid with a yield of $82 \%$.

${ }^{1} \mathrm{H}-\mathrm{NMR}\left(300 \mathrm{MHz}, \mathrm{CDCl}_{3}\right): \delta(\mathrm{ppm})=8.14-8.00(\mathrm{~m}, 2 \mathrm{H}), 7.51-7.31(\mathrm{~m}, 4 \mathrm{H}), 5.25(\mathrm{dd}, \mathrm{J}=7.1,4.9$ $\mathrm{Hz}, 1 \mathrm{H}$ ), 3.01 (ddd, J = 16.2, 8.7, $4.9 \mathrm{~Hz}, 1 \mathrm{H}$ ), 2.72 (ddd, J = 16.2, 8.5, $5.9 \mathrm{~Hz}, 1 \mathrm{H}$ ), 2.46 (dddd, J = 13.4, 8.4, 7.1, 5.0 Hz, 1H), 2.32 (s, 3H), 2.06 (dddd, J = 13.5, 8.8, 5.9, 4.9 Hz, 1H), $0.99(\mathrm{~s}, 9 \mathrm{H}), 0.28$ $(\mathrm{s}, 3 \mathrm{H}), 0.21(\mathrm{~s}, 3 \mathrm{H}) .{ }^{13} \mathrm{C}-\mathrm{NMR}\left(75 \mathrm{MHz}, \mathrm{CDCl}_{3}\right) \delta(\mathrm{ppm})=164.0,156.2,143.9,139.9,134.2,128.5$, $128.3,126.8,120.1,76.4,33.9,26.0,25.8,18.8,18.6,-4.1,-4.6$.

\subsubsection{7. (S)-7-((tert-butyldimethylsilyl)oxy)-2-(4-methoxyphenyl)-4-methyl-6,7- dihydro-5H-cyclopenta[b]pyridine}<smiles>COc1ccc(-c2cc(C)c3nc2CCC3O[Si](C)(C)C(C)(C)C)cc1</smiles>

The reaction was performed once in our laboratory ( $2 \mathrm{mmol}$ scale) according to the above described procedure (1.1.2.). The product could be isolated as white solid with a yield of $82 \%$.

${ }^{1} \mathrm{H}-\mathrm{NMR}\left(300 \mathrm{MHz}, \mathrm{CDCl}_{3}\right): \delta(\mathrm{ppm})=8.05-7.99(\mathrm{~m}, 2 \mathrm{H}), 7.38-7.34(\mathrm{~m}, 1 \mathrm{H}), 7.00-6.94(\mathrm{~m}, 2 \mathrm{H})$, $5.29-5.19(\mathrm{~m}, 1 \mathrm{H}), 3.86(\mathrm{~s}, 3 \mathrm{H}), 3.05-2.92(\mathrm{~m}, 1 \mathrm{H}), 2.76-2.63(\mathrm{~m}, 1 \mathrm{H}), 2.53-2.39(\mathrm{~m}, 1 \mathrm{H}), 2.34$ $-2.24(\mathrm{~m}, 3 \mathrm{H}), 2.12-1.98(\mathrm{~m}, 1 \mathrm{H}), 1.00(\mathrm{~s}, 9 \mathrm{H}), 0.29(\mathrm{~s}, 3 \mathrm{H}), 0.21(\mathrm{~s}, 3 \mathrm{H}) .{ }^{13} \mathrm{C}-\mathrm{NMR}\left(75 \mathrm{MHz}, \mathrm{CDCl}_{3}\right)$ $\delta(\mathrm{ppm})=164.0,160.1,156.1,143.9,133.5,132.7,128.1,119.4,114.0,76.5,55.4,34.0,26.2,25.9$, $18.9,18.8,-4.0,-4.4$.

\subsubsection{8. (S)-2-(4-(trifluoromethyl)phenyl)-7-((tert-butyldimethylsilyl)oxy)-4- methyl-6,7-dihydro-5H-cyclopenta[b]pyridine \\ according to the above described procedure (1.1.2.). The product
could be isolated as white solid with a yield of $80 \%$.} ${ }^{1} \mathrm{H}-\mathrm{NMR}\left(300 \mathrm{MHz}, \mathrm{CDCl}_{3}\right): \delta(\mathrm{ppm})=8.17(\mathrm{~d}, J=8.0 \mathrm{~Hz}, 2 \mathrm{H}), 7.74-7.62(\mathrm{~m}, 2 \mathrm{H}), 7.49-7.42(\mathrm{~s}$, 1H), 5.25 (dd, J = 7.2, 5.2 Hz, 1H), 3.01 (ddd, J = 16.4, 8.8, $4.7 \mathrm{~Hz}, 1 \mathrm{H}$ ), 2.73 (ddd, $J=16.3,8.4,6.2$ $\mathrm{Hz}, 1 \mathrm{H}$ ), 2.48 (dddd, $J=13.2,8.5,7.2,4.7 \mathrm{~Hz}, 1 \mathrm{H}$ ), 2.33 (d, $3 \mathrm{H}$ ), 2.06 (dddd, $J=13.3,8.8,6.3,5.2 \mathrm{~Hz}$, 1H), 0.99 (s, 9H), 0.27 (s, 3H), 0.21 (s, 3H). ${ }^{13} \mathrm{C}-\mathrm{NMR}\left(75 \mathrm{MHz}, \mathrm{CDCl}_{3}\right) \delta(\mathrm{ppm})=164.4,154.7,144.2$, 143.2, 135.1, 133.7, 130.3, 129.9, 126.9, 126.2, 125.4, 122.6, 120.6, 76.2, 33.8, 26.0, 25.8, 18.8, $18.6,-4.2,-4.6$ 
SI: Application of Crabtree/Pfaltz-type iridium complexes for the catalyzed asymmetric hydrogenation of an agrochemical building block

2.1.2.9. $S$ )-2-(4-(tert-butyl)phenyl)-7-((tert-butyldimethylsilyl)oxy)-4-methyl-6,7dihydro-5H-cyclopenta[b]pyridine

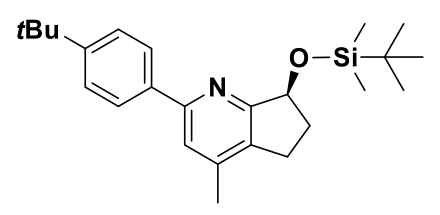

The reaction was performed twice in our laboratory $(2 \mathrm{mmol}$ scale) according to the above described procedure (1.1.2.). The product could be isolated as white solid with a yield of $50 \%$ in the first attempt and with a yield of $64 \%$ in the second.

${ }^{1} \mathrm{H}-\mathrm{NMR}\left(300 \mathrm{MHz}, \mathrm{CDCl}_{3}\right) \delta(\mathrm{ppm})=8.13-7.99(\mathrm{~m}, 2 \mathrm{H}), 7.61-7.48(\mathrm{~m}, 2 \mathrm{H}), 7.45-7.42(\mathrm{~m}, 1 \mathrm{H})$, $5.29(\mathrm{dd}, \mathrm{J}=7.1,4.8 \mathrm{~Hz}, 1 \mathrm{H}), 3.11-2.94(\mathrm{~m}, 1 \mathrm{H}), 2.82-2.64(\mathrm{~m}, 1 \mathrm{H}), 2.59-2.39(\mathrm{~m}, 1 \mathrm{H}), 2.33(\mathrm{~s}$, 3H), $2.21-2.01(\mathrm{~m}, 1 \mathrm{H}), 1.42(\mathrm{~s}, 9 \mathrm{H}), 1.06(\mathrm{~s}, 9 \mathrm{H}), 0.35(\mathrm{~s}, 3 \mathrm{H}), 0.28(\mathrm{~s}, 3 \mathrm{H}) .{ }^{13} \mathrm{C}-\mathrm{NMR}\left(75 \mathrm{MHz}, \mathrm{CDCl}_{3}\right)$ $\delta(\mathrm{ppm})=164.0,156.3,151.4,143.8,137.2$, 133.9, 126.6, 125.6, 120.0, 76.5, 34.7, 33.9, 31.5, 26.2, $25.9,18.9,18.7,-4.0,-4.5$.

\subsubsection{0. (S)- 2-(anthracen-9-yl)-7-((tert-butyldimethylsilyl)oxy)-4-methyl-6,7-} dihydro-5H-cyclopenta[b]pyridine

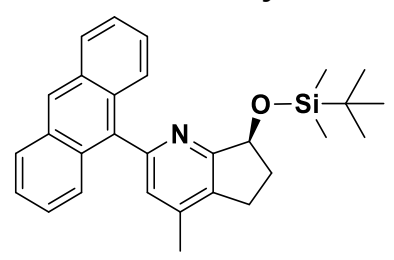

The reaction was performed once in our laboratory $(2 \mathrm{mmol}$ scale) according to the above described procedure (1.1.2.). A very impurity product was obtained as yellowish solid. This mixture was used for next step without further purification.

2.1.2.11. (S)-7-((tert-butyldimethylsilyl)oxy)-4-methyl-2-(naphthalene-1-yl)-6,7dihydro-5H-cyclopenta[b]pyridine

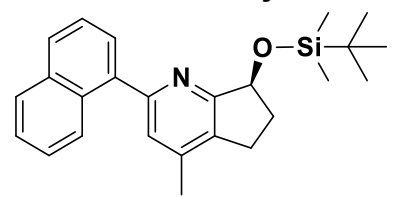

The reaction was performed once in our laboratory $(2 \mathrm{mmol}$ scale) according to the above described procedure (1.1.2.). The product could be isolated as white solid with a yield of $85 \%$.

${ }^{1} \mathrm{H}-\mathrm{NMR}\left(300 \mathrm{MHz}, \mathrm{CDCl}_{3}\right) \delta(\mathrm{ppm})=8.47-8.26(\mathrm{~m}, 1 \mathrm{H}), 7.95-7.82(\mathrm{~m}, 2 \mathrm{H}), 7.66-7.59(\mathrm{~m}, 1 \mathrm{H})$, $7.57-7.40(\mathrm{~m}, 3 \mathrm{H}), 7.27(\mathrm{~s}, 1 \mathrm{H}), 5.37-5.17(\mathrm{~m}, 1 \mathrm{H}), 3.18-3.03(\mathrm{~m}, 1 \mathrm{H}), 2.87-2.73(\mathrm{~m}, 1 \mathrm{H}), 2.58$ $-2.40(\mathrm{~m}, 1 \mathrm{H}), 2.39-2.31(\mathrm{~m}, 3 \mathrm{H}), 2.20-2.04(\mathrm{~m}, 1 \mathrm{H}), 0.97(\mathrm{~s}, 9 \mathrm{H}), 0.22(\mathrm{~s}, 3 \mathrm{H}), 0.18(\mathrm{~s}, 3 \mathrm{H}) .{ }^{13} \mathrm{C}-$ $\operatorname{NMR}\left(75 \mathrm{MHz}, \mathrm{CDCl}_{3}\right) \delta(\mathrm{ppm})=134.1,131.5,128.6,128.2,127.7,126.8,126.1,125.8,125.3,125.2$, $76.5,34.0,26.2,26.1,18.9,18.6,-4.0,-4.4$.

2.1.2.12. (S)-7-((tert-butyldimethylsilyl)oxy)-2-(3,5-dimethylphenyl)-4-methyl-6,7dihydro-5H-cyclopenta[b]pyridine

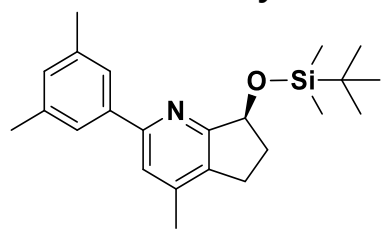

The reaction was performed once in our laboratory ( $2 \mathrm{mmol}$ scale) according to the above described procedure (1.1.2.). The product could be isolated as white solid with a yield of $51 \%$. 


\section{SI: Application of Crabtree/Pfaltz-type iridium complexes for the catalyzed asymmetric hydrogenation of an agrochemical building block}

${ }^{1} \mathrm{H}-\mathrm{NMR}\left(300 \mathrm{MHz}, \mathrm{CDCl}_{3}\right) \delta(\mathrm{ppm})=7.70(\mathrm{dd}, \mathrm{J}=1.1,0.6 \mathrm{~Hz}, 1 \mathrm{H}), 7.41(\mathrm{~s}, 1 \mathrm{H}), 7.02(\mathrm{~s}, 1 \mathrm{H}), 5.25(\mathrm{t}$, $J=6.1 \mathrm{~Hz}, 1 \mathrm{H}$ ), $3.00(\mathrm{ddd}, J=16.2,8.7,4.9 \mathrm{~Hz}, 1 \mathrm{H}), 2.71(\mathrm{ddd}, J=16.0,8.4,5.8 \mathrm{~Hz}, 1 \mathrm{H}), 2.53-2.41$ $(\mathrm{m}, 1 \mathrm{H}), 2.39(\mathrm{~d}, J=0.7 \mathrm{~Hz}, 6 \mathrm{H}), 2.31(\mathrm{~s}, 3 \mathrm{H}), 2.05$ (dddd, $J=13.6,8.8,5.9,4.9 \mathrm{~Hz}, 1 \mathrm{H}), 1.00(\mathrm{~s}, 9 \mathrm{H})$, $0.27(\mathrm{~s}, 3 \mathrm{H}), 0.22(\mathrm{~s}, 3 \mathrm{H}) .{ }^{13} \mathrm{C}-\mathrm{NMR}\left(75 \mathrm{MHz}, \mathrm{CDCl}_{3}\right) \delta(\mathrm{ppm})=163.9,156.4,143.7,137.9,133.9$, $130.0,124.8,120.2,100.0,76.4,33.9,26.0,25.8,21.5,18.8,18.7,-4.2,-4.5$.

\subsubsection{3. (S)-7-((tert-butyldimethylsilyl)oxy)-2-(2,6-dimethylphenyl)-4-methyl-6,7- dihydro-5H-cyclopenta[b]pyridine}

The reaction was performed once in our laboratory ( $2 \mathrm{mmol}$ scale). (S)-7-((tert-butyldimethylsilyl)oxy)-2-chloro-4-methyl-6,7-dihydro-

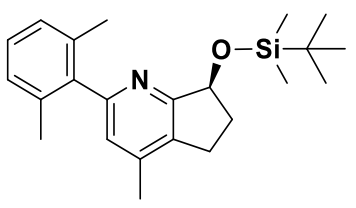
$5 \mathrm{H}$-cyclopenta $[b]$ pyridine $(2.0 \mathrm{mmol}),(2,6$-dimethylphenyl)boronic acid (1.15 equiv.), Nolan NHC catalyst ( 2 mol\%), isopropanol $(5 \mathrm{~mL})$, and $\mathrm{NaOH}$ (3.0 equiv, $4 \mathrm{M}$ in $\mathrm{H}_{2} \mathrm{O}$ ) were used. The reaction was performed in a Schlenk pressure tube, and stirred at $115^{\circ} \mathrm{C}$ for $48 \mathrm{~h}$. The product could be isolated as white solid with a yield of $82 \%$.

${ }^{1} \mathrm{H}-\mathrm{NMR}\left(300 \mathrm{MHz}, \mathrm{CDCl}_{3}\right): \delta(\mathrm{ppm})=7.22-7.01(\mathrm{~m}, 3 \mathrm{H}), 6.88(\mathrm{~s}, 1 \mathrm{H}), 3.07$ (ddd, $J=15.4,8.0,6.4$ $\mathrm{Hz}, 1 \mathrm{H}), 2.77(\mathrm{ddd}, J=16.1,8.6,4.1 \mathrm{~Hz}, 1 \mathrm{H}), 2.40(\mathrm{ddt}, J=13.3,8.6,6.6 \mathrm{~Hz}, 1 \mathrm{H}), 2.30(\mathrm{~s}, 3 \mathrm{H}), 2.13-$ $2.03(\mathrm{~m}, 7 \mathrm{H}), 0.90(\mathrm{~s}, 9 \mathrm{H}), 0.18(\mathrm{~s}, 3 \mathrm{H}), 0.10(\mathrm{~s}, 3 \mathrm{H})$.

\subsubsection{4. (S)-7-((tert-butyldimethylsilyl)oxy)-2-mesityl-4-methyl-6,7-dihydro-5H- cyclopenta[b]pyridine}

The reaction was performed once in our laboratory ( $2 \mathrm{mmol}$ scale).

(S)-7-((tert-butyldimethylsilyl)oxy)-2-chloro-4-methyl-6,7-dihydro$5 H$-cyclopenta $[b]$ pyridine $(2.0 \mathrm{mmol})$, mesitylboronic acid $(1.15$<smiles>Cc1cc(C)c(-c2cc(C)c3c(n2)CCC3O[Si](C)(C)C(C)(C)C)c(C)c1</smiles>
equiv.), Nolan $\mathrm{NHC}$ catalyst ( $2 \mathrm{~mol} \%$ ), isopropanol $(5 \mathrm{~mL})$, and $\mathrm{NaOH}$ (3.0 equiv, $4 \mathrm{M}$ in $\mathrm{H}_{2} \mathrm{O}$ ) were used. The reaction was performed in a Schlenk pressure tube, and stirred at $115{ }^{\circ} \mathrm{C}$ for $48 \mathrm{~h}$. The product could be isolated as white solid with a yield of $82 \%$. The quality of the product was checked by GC-MS as well as TLC analysis and was used directely for the next step. 
SI: Application of Crabtree/Pfaltz-type iridium complexes for the catalyzed asymmetric hydrogenation of an agrochemical building block

\subsubsection{5. (R)-7-((tert-butyldimethylsilyl)oxy)-2-methyl-6,7-dihydro-5H-} cyclopenta[b]pyridine

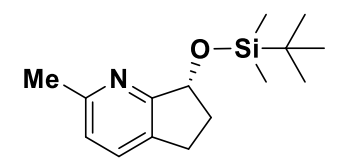

The reaction was performed once in our laboratory $(2 \mathrm{mmol}$ scale).

(R)-7-((tert-butyldimethylsilyl)oxy)-2-chloro-6,7-dihydro-5H-

cyclopenta[b]pyridine $(2.0 \mathrm{mmol})$, trimethylboroxine (1.5 equiv.), (dppf) $\mathrm{PdCl}_{2} \cdot \mathrm{DCM}(5 \mathrm{~mol} \%), \mathrm{K}_{2} \mathrm{CO}_{3}$ (3.0 equiv) and 1,4-dioxane $(5 \mathrm{~mL}$ ) were used. The reaction was performed in a Schlenk pressure tube, and stirred at $120{ }^{\circ} \mathrm{C}$ for $20 \mathrm{~h}$. The product could be isolated as colorless oil a yield of $95 \%$. The quality of the product was checked by GC-MS as well as TLC analysis and was used directly for the next step.

2.1.2.16. (S)-7-((tert-butyldimethylsilyl)oxy)-2-(3,5-di-tert-butyl-4-methoxyphenyl)4-methyl-6,7-dihydro-5H-cyclopenta[b]pyridine

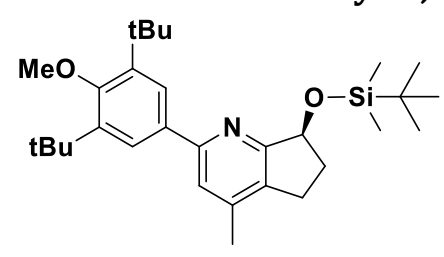

The reaction was performed once in our laboratory $(1 \mathrm{mmol}$ scale) according to the above described procedure (1.1.2.). The product could be isolated as white solid with a yield of $69 \%$. The quality of the product was checked by GC-MS as well as TLC analysis and was used directly for the next step.

2.1.2.17. (S)-7-((tert-butyldimethylsilyl)oxy)-4-methyl-2-(2,4,6-triisopropylphenyl)6,7-dihydro-5H-cyclopenta[b]pyridine

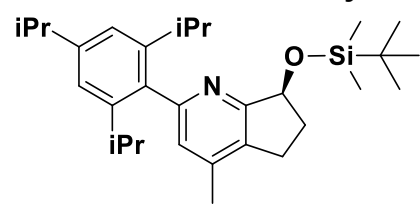

The reaction was performed once in our laboratory ( $1 \mathrm{mmol}$ scale).

(S)-2-chloro-4-methyl-6,7-dihydro-5H-cyclopenta[b]pyridin-7-ol

(1.0 mmol), (2,4,6-triisopropylphenyl)boronic acid (1.2 equiv.), $\mathrm{Pd}\left(\mathrm{PPh}_{3}\right)_{4}(15 \mathrm{~mol} \%), \mathrm{K}_{3} \mathrm{PO}_{4}$ (5.0 equiv), $\mathrm{H}_{2} \mathrm{O}(1.0 \mathrm{~mL})$ and DME (5.0 $\mathrm{mL}$ ) were used. The reaction was performed in a Schlenk pressure tube, and stirred at $110^{\circ} \mathrm{C}$ for $24 \mathrm{~h}$. The product could be isolated as white solid a yield of $66 \%$. The quality of the product was checked by GC-MS as well as TLC analysis and was used directly for the next step. 


\section{SI: Application of Crabtree/Pfaltz-type iridium complexes for the catalyzed asymmetric hydrogenation of an agrochemical building block}

\subsubsection{8. (S)-2-(4-(tert-butyl)-2,6-dim ethylphenyl)-7-((tert-butyldimethylsilyl)oxy)- 4-methyl-6,7-dihydro-5H-cyclopenta[b]pyridine

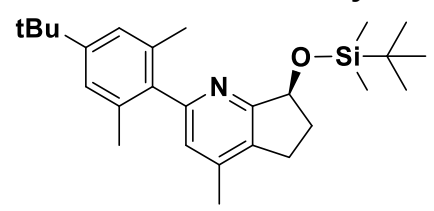 \\ The reaction was performed once in our laboratory ( $2 \mathrm{mmol}$ scale). \\ (S)-2-chloro-4-methyl-6,7-dihydro-5H-cyclopenta [b]pyridin-7-ol \\ $(2.0 \mathrm{mmol})$, the above synthesized impure (4-(tert-butyl)-2,6- dimethylphenyl)boronic acid ( $824 \mathrm{mg}, 2.0$ equiv), Nolan NHC catalyst (2 mol\%), isopropanol $\left(5 \mathrm{~mL}\right.$ ), and $\mathrm{NaOH}\left(3.0\right.$ equiv, $4 \mathrm{M}$ in $\mathrm{H}_{2} \mathrm{O}$ ) were used. The reaction was performed in a Schlenk pressure tube, and stirred at $115^{\circ} \mathrm{C}$ for $24 \mathrm{~h}$. The product could be isolated as white solid with a yield of $85 \%$. The quality of the product was checked by GC- MS as well as TLC analysis and was used directly for the next step.}

\subsubsection{Synthesis of pyridyl alcohols by deprotection of OTBDMS ether}

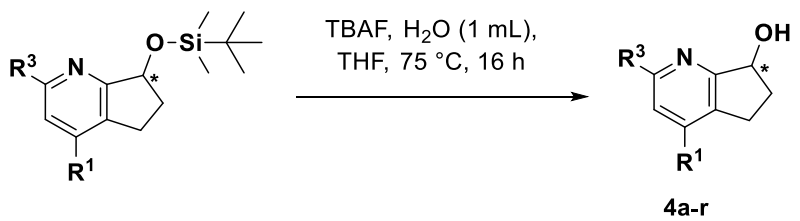

Literature: $\quad$ D. H. Woodmansee, M.-A. Müller, M. Neuburger, A. Pfaltz Chem. Sci., 2010, 1, 72.

Procedure: The 2-arylpyridyl silyl ether $(1 \mathrm{mmol})$ was taken up in $25 \mathrm{~mL}$ of dry THF. Tetrabutylammonium fluoride ( $3 \mathrm{~mL}$ of a $1 \mathrm{M}$ solution THF) and $1 \mathrm{~mL}$ of degassed $\mathrm{H}_{2} \mathrm{O}$ were added via syringe. The reaction was stirred at $75{ }^{\circ} \mathrm{C}$ overnight. The reaction was monitored by TLC, if the conversion was incomplete an additional amounts of TBAF $(1 \mathrm{~mL})$ and $\mathrm{H}_{2} \mathrm{O}(0.5 \mathrm{~mL})$ were added and the heating continued upon compilation of the deprotection. The heating was stopped when full conversion was achieved by pouring the reaction after cooling to room temperature into an appropriately sized separatory funnel, addition and washing of the flask into the funnel with $75 \mathrm{~mL}$ of EtOAc and extraction with water $(3 \times 50 \mathrm{~mL})$. The organic layer was washed with brine $(2 \times 25 \mathrm{~mL})$. The organic layer was dried over $\mathrm{MgSO}_{4}$, filtered and concentrated at the rotary evaporator. The crude was purified by column chromatography on a flash column with a heptane/EtOAc: $3 / 1 \rightarrow 1 / 1$.

\subsubsection{1. $\quad(R)$-2-phenyl-6,7-dihydro-5H-cyclopenta[b]pyridin-7-ol (4a)}

The reaction was performed twice in our laboratory according to the<smiles>O[C@@H]1CCc2ccc(-c3ccccc3)nc21</smiles>
above described procedure (1.1.3.). In the first run ( $0.86 \mathrm{mmol}$ scale) the product could be isolated as a white solid in $92 \%$ yield. In the second run $(5.85 \mathrm{mmol})$ a slightly lower yield of $82 \%$ was obtained. 


\section{SI: Application of Crabtree/Pfaltz-type iridium complexes for the catalyzed asymmetric hydrogenation of an agrochemical building block}

${ }^{1} \mathrm{H}-\mathrm{NMR}\left(300 \mathrm{MHz}, \mathrm{CDCl}_{3}\right): \delta(\mathrm{ppm})=8.03-7.87(\mathrm{~m}, 2 \mathrm{H}), 7.71-7.53(\mathrm{~m}, 2 \mathrm{H}), 7.53-7.33(\mathrm{~m}, 3 \mathrm{H})$, $5.33-5.18(\mathrm{~m}, 1 \mathrm{H}), 3.32(\mathrm{~s}, 1 \mathrm{H}), 3.13-2.97(\mathrm{~m}, 1 \mathrm{H}), 2.96-2.76(\mathrm{~m}, 1 \mathrm{H}), 2.68-2.48(\mathrm{~m}, 1 \mathrm{H}), 2.18-$ $1.98(\mathrm{~m}, 1 \mathrm{H}) .{ }^{13} \mathrm{C}-\mathrm{NMR}\left(75 \mathrm{MHz}, \mathrm{CDCl}_{3}\right) \delta(\mathrm{ppm})=164.8,156.3,139.3,134.7,133.6,128.6,126.9$, $120.9,74.4,32.9,27.1,19.7$.

\subsubsection{2. (R)-2-(4-methoxyphenyl)-6,7-dihydro-5H-cyclopenta[b]pyridin-7-ol (4c)} The reaction was performed twice in our laboratory according to the<smiles>COc1ccc(-c2ccc3c(n2)[C@@H](O)CC3)cc1</smiles>
above described procedure (1.1.3.). In the first run (1 mmol scale) the product could be isolated as a white solid in $80 \%$ yield. In the second run ( $3 \mathrm{mmol}$ scale) gave a lower yield of $62 \%$.

${ }^{1} \mathrm{H}-\mathrm{NMR}\left(300 \mathrm{MHz}, \mathrm{CDCl}_{3}\right): \delta(\mathrm{ppm})=7.98-7.86(\mathrm{~m}, 2 \mathrm{H}), 7.67-7.47(\mathrm{~m}, 2 \mathrm{H}), 7.04-6.89(\mathrm{~m}, 2 \mathrm{H})$, $5.30-5.18(\mathrm{~m}, 1 \mathrm{H}), 3.85(\mathrm{~s}, 3 \mathrm{H}), 3.43(\mathrm{~s}, 1 \mathrm{H}), 3.08-2.95(\mathrm{~m}, 1 \mathrm{H}), 2.90-2.75(\mathrm{~m}, 1 \mathrm{H}), 2.64-2.46$ (m, 1H), $2.11-1.96(\mathrm{~m}, 1 \mathrm{H}) .{ }^{13} \mathrm{C}-\mathrm{NMR}(75 \mathrm{MHz}, \mathrm{CDCl}) \delta(\mathrm{ppm})=164.3,160.3,156.1,134.1,133.9$, $131.8,128.3,119.4,114.1,74.9,55.4,33.0,27.2$.

\subsubsection{3. (R)-2-(anthracen-9-yl)-6,7-dihydro-5H-cyclopenta[b]pyridin-7-ol (4d)}<smiles>O[C@H]1CCc2ccc(-c3c4ccccc4cc4ccccc34)nc21</smiles>
The reaction was performed twice in our laboratory according to the above described procedure (1.1.3.). In the first run (0.86 mmol scale) the product could be isolated as an orange solid in $43 \%$ yield. In the second run ( $3 \mathrm{mmol}$ ) a slightly higher yield of $50 \%$.

${ }^{1} \mathrm{H}-\mathrm{NMR}\left(300 \mathrm{MHz}, \mathrm{CDCl}_{3}\right): \delta(\mathrm{ppm})=8.55(\mathrm{~s}, 1 \mathrm{H}), 8.17-7.98(\mathrm{~m}, 2 \mathrm{H}), 7.79(\mathrm{~d}, \mathrm{~J}=7.7 \mathrm{~Hz}, 1 \mathrm{H}), 7.64$ $-7.53(\mathrm{~m}, 2 \mathrm{H}), 7.51-7.42(\mathrm{~m}, 2 \mathrm{H}), 7.42-7.31(\mathrm{~m}, 3 \mathrm{H}), 5.37-5.26(\mathrm{~m}, 1 \mathrm{H}), 3.43-3.09(\mathrm{~m}, 2 \mathrm{H}), 3.03$ $-2.90(\mathrm{~m}, 1 \mathrm{H}), 2.69-2.50(\mathrm{~m}, 1 \mathrm{H}), 2.22-2.01(\mathrm{~m}, 1 \mathrm{H}) .{ }^{13} \mathrm{C}-\mathrm{NMR}\left(75 \mathrm{MHz}, \mathrm{CDCl}_{3}\right) \delta(\mathrm{ppm})=164.9$, $156.5,135.2$, 134.5, 133.7, 131.4, 131.4, 130.2, 128.5, 128.4, 127.7, 126.1, 126.1, 126.0, 125.9, $125.9,125.2,125.1,74.8,32.6,27.5$.

\subsubsection{4. (R)-2-(4-tert-butylphenyl)-6,7-dihydro-5H-cyclopenta[b]pyridin-7-ol (4e) The reaction was performed twice in our laboratory according to the<smiles>CC(C)(C)c1ccc(-c2ccc3c(n2)C(O)CC3)cc1</smiles> above described procedure (1.1.3.). In the first run (1 mmol scale) the product could be isolated as a white solid in $74 \%$ yield. In the second run ( $3 \mathrm{mmol}$ ) a slightly higher yield of $79 \%$ was obtained.}

${ }^{1} \mathrm{H}-\mathrm{NMR}\left(300 \mathrm{MHz}, \mathrm{CDCl}_{3}\right): \delta(\mathrm{ppm})=7.94-7.80(\mathrm{~m}, 2 \mathrm{H}), 7.62-7.51(\mathrm{~m}, 2 \mathrm{H}), 7.50-7.43(\mathrm{~m}, 2 \mathrm{H})$, $5.31-5.19(\mathrm{~m}, 1 \mathrm{H}), 3.81(\mathrm{~s}, 1 \mathrm{H}), 3.08-2.93(\mathrm{~m}, 1 \mathrm{H}), 2.92-2.76(\mathrm{~m}, 1 \mathrm{H}), 2.62-2.46(\mathrm{~m}, 1 \mathrm{H}), 2.16-$ $1.96(\mathrm{~m}, 1 \mathrm{H}), 1.36(\mathrm{~s}, 9 \mathrm{H}) .{ }^{13} \mathrm{C}-\mathrm{NMR}\left(75 \mathrm{MHz}, \mathrm{CDCl}_{3}\right) \delta(\mathrm{ppm})=164.7,156.6,151.9,136.7,134.6$, $133.8,126.8,125.7,120.0,74.9,34.8,33.1,31.4,27.4$. 
SI: Application of Crabtree/Pfaltz-type iridium complexes for the catalyzed asymmetric hydrogenation of an agrochemical building block

2.1.3.5. (S)-2-(4-fluorophenyl))-4-methyl-6,7-dihydro-5H-cyclopenta[b]pyridin-7ol (4f)<smiles>Cc1cc(-c2ccc(F)cc2)nc2c1CCC2O</smiles>

The reaction was performed once in our laboratory $(2 \mathrm{mmol}$ scale) according to the above described procedure (1.1.3.). The product could be isolated as white solid with a yield of $84 \%$.

${ }^{1} \mathrm{H}-\mathrm{NMR}\left(300 \mathrm{MHz}, \mathrm{CDCl}_{3}\right): \delta(\mathrm{ppm})=7.95-7.83(\mathrm{~m}, 2 \mathrm{H}), 7.37-7.29(\mathrm{~m}, 1 \mathrm{H}), 7.00-6.91(\mathrm{~m}, 2 \mathrm{H})$, $5.27-5.17(\mathrm{~m}, 1 \mathrm{H}), 3.84(\mathrm{~s}, 3 \mathrm{H}), 3.60(\mathrm{~s}, 1 \mathrm{H}), 3.05-2.88(\mathrm{~m}, 1 \mathrm{H}), 2.81-2.64(\mathrm{~m}, 1 \mathrm{H}), 2.62-2.46$ $(\mathrm{m}, 1 \mathrm{H}), 2.35-2.26(\mathrm{~m}, 3 \mathrm{H}), 2.11-1.96(\mathrm{~m}, 1 \mathrm{H}) .{ }^{13} \mathrm{C}-\mathrm{NMR}\left(75 \mathrm{MHz}, \mathrm{CDCl}_{3}\right) \delta(\mathrm{ppm})=163.9,160.1$, $156.4,144.5,133.5,132.2,128.2,120.4,114.0,75.1,66.7,55.4,32.5,25.8,18.8$.

\subsubsection{6. (S)-4-methyl-2-phenyl-6,7-dihydro-5H-cyclopenta[b]pyridin-7-ol (4b)}<smiles>Cc1cc(-c2ccccc2)nc2c1CCC2O</smiles>

The reaction was performed in our laboratory ( $2 \mathrm{mmol}$ scale) according to the above described procedure (1.1.3.). The product could be isolated as white solid with a yield of $91 \%$.

${ }^{1} \mathrm{H}-\mathrm{NMR}\left(400 \mathrm{MHz}, \mathrm{CDCl}_{3}\right): \delta(\mathrm{ppm})=7.96-7.88(\mathrm{~m}, 2 \mathrm{H}), 7.48-7.32(\mathrm{~m}, 4 \mathrm{H}), 5.24(\mathrm{dd}, J=7.4,5.8$ $\mathrm{Hz}, 1 \mathrm{H}$ ), 3.55 (s, 1H), 2.97 (ddd, $J=16.3,8.9,4.0 \mathrm{~Hz}, 1 \mathrm{H}$ ), $2.81-2.65(\mathrm{~m}, 1 \mathrm{H}), 2.55$ (dddd, $J=13.4$, 8.4, 7.4, $4.1 \mathrm{~Hz}, 1 \mathrm{H}$ ), 2.33 (s, 3H), 2.05 (dddd, $J=13.4,9.0,6.8,5.8 \mathrm{~Hz}, 1 \mathrm{H}) .{ }^{13} \mathrm{C}-\mathrm{NMR}\left(100 \mathrm{MHz}, \mathrm{CDCl}_{3}\right)$ $\delta(\mathrm{ppm})=164.0,156.8,144.6,139.5,134.2,128.6,128.6,127.0,121.1,75.1,32.5,25.9,18.8$.

\subsubsection{7. (S)-2-(4-methoxyphenyl)-4-methyl-6,7-dihydro-5H-cyclopenta[b]pyridin-7-} ol (4g)<smiles>COc1ccc(-c2cc(C)c3nc2C(O)CC3)cc1</smiles>

The reaction was performed once in our laboratory $(2 \mathrm{mmol}$ scale) according to the above described procedure (1.1.3.). The product could be isolated as white solid with a yield of $94 \%$.

${ }^{1} \mathrm{H}-\mathrm{NMR}\left(300 \mathrm{MHz}, \mathrm{CDCl}_{3}\right): \delta(\mathrm{ppm})=7.96-7.83(\mathrm{~m}, 2 \mathrm{H}), 7.35-7.30(\mathrm{~m}, 1 \mathrm{H}), 7.00-6.90(\mathrm{~m}, 2 \mathrm{H})$, $5.28-5.16(\mathrm{~m}, 1 \mathrm{H}), 3.84(\mathrm{~s}, 3 \mathrm{H}), 3.60(\mathrm{~s}, 1 \mathrm{H}), 3.04-2.88(\mathrm{~m}, 1 \mathrm{H}), 2.81-2.65(\mathrm{~m}, 1 \mathrm{H}), 2.62-2.47$ $(\mathrm{m}, 1 \mathrm{H}), 2.13-1.95(\mathrm{~m}, 1 \mathrm{H}) .{ }^{13} \mathrm{C}-\mathrm{NMR}\left(75 \mathrm{MHz}, \mathrm{CDCl}_{3}\right) \delta(\mathrm{ppm})=164.0,160.3,156.5,144.6,133.6$, $132.3,128.3,120.5,114.1,75.2,55.5,32.6,26.0,18.9$. 
SI: Application of Crabtree/Pfaltz-type iridium complexes for the catalyzed asymmetric hydrogenation of an agrochemical building block

2.1.3.8. (S)- 4-methyl-2-(4-(trifluoromethyl)phenyl)-4-mehyl-6,7-dihydro-5Hcyclopenta[b]pyridin-7-ol (4h)

(n)

The reaction was performed once in our laboratory $(1.55 \mathrm{mmol}$ scale) according to the above described procedure (1.1.3.). The product could be isolated as white solid with a yield of $95 \%$.

${ }^{1} \mathrm{H}-\mathrm{NMR}\left(400 \mathrm{MHz}, \mathrm{CDCl}_{3}\right): \delta(\mathrm{ppm})=8.03-7.97(\mathrm{~m}, 2 \mathrm{H}), 7.68-7.62(\mathrm{~m}, 2 \mathrm{H}), 7.39(\mathrm{~s}, 1 \mathrm{H}), 5.27-$ $5.21(\mathrm{~m}, 1 \mathrm{H}), 4.20(\mathrm{~s}, 1 \mathrm{H}), 3.04-2.92(\mathrm{~m}, 1 \mathrm{H}), 2.80-2.70(\mathrm{~m}, 1 \mathrm{H}), 2.61-2.50(\mathrm{~m}, 1 \mathrm{H}), 2.33(\mathrm{~s}, 3 \mathrm{H})$, $2.12-2.00(\mathrm{~m}, 1 \mathrm{H}) .{ }^{13} \mathrm{C}-\mathrm{NMR}\left(75 \mathrm{MHz}, \mathrm{CDCl}_{3}\right)=164.5,155.1,145.0,142.8,135.3,130.4$ (q, $J=32.4$ $\mathrm{Hz}), 127.7,127.2,125.5(q, J=3.7 \mathrm{~Hz}), 121.5,74.9,32.4,25.9,18.8$.

2.1.3.9. (S)-2-(4-(tert-butyl)phenyl)-4-methyl-6,7-dihydro-5Hcyclopenta[b]pyridin-7-ol (4i)

${ }^{t B u}$ The reaction was performed once in our laboratory ( $2 \mathrm{mmol}$ scale) according to the above described procedure (1.1.3.). The product could be isolated as beige solid with a yield of $84 \%$.

${ }^{1} \mathrm{H}-\mathrm{NMR}\left(300 \mathrm{MHz}, \mathrm{CDCl}_{3}\right): \delta(\mathrm{ppm})=7.89-7.81(\mathrm{~m}, 2 \mathrm{H}), 7.49-7.42(\mathrm{~m}, 2 \mathrm{H}), 7.38-7.34(\mathrm{~m}, 1 \mathrm{H})$, $5.24(\mathrm{dd}, \mathrm{J}=7.3,5.8 \mathrm{~Hz}, 1 \mathrm{H}), 3.88(\mathrm{~s}, 1 \mathrm{H}), 2.94(\mathrm{ddd}, \mathrm{J}=16.2,8.9,4.1 \mathrm{~Hz}, 1 \mathrm{H}), 2.81-2.63(\mathrm{~m}, 1 \mathrm{H})$, $2.63-2.45(\mathrm{~m}, 1 \mathrm{H}), 2.31(\mathrm{~d}, \mathrm{~J}=0.7 \mathrm{~Hz}, 3 \mathrm{H}), 1.36(\mathrm{~s}, 9 \mathrm{H}) .{ }^{13} \mathrm{C}-\mathrm{NMR}\left(75 \mathrm{MHz}, \mathrm{CDCl}_{3}\right)=164.0,156.8$, $151.8,144.8,136.7,134.1,126.8,125.7,121.0,75.1,34.8,32.6,31.4,26.0,18.9$.

2.1.3.10. (S)-2-(anthracen-9-yl)-4-methyl-6,7-dihydro-5H-cyclopenta[b]pyridin-7-ol (4j)<smiles>Cc1cc(-c2c3ccccc3cc3ccccc23)nc2c1CCC2O</smiles>

The reaction was performed once in our laboratory using the above obtained mixture, TBAF $(6.0 \mathrm{mmol}), \operatorname{THF}(50 \mathrm{~mL})$ and $\mathrm{H}_{2} \mathrm{O}(2 \mathrm{~mL})$, according to the above described procedure (1.1.3.). The product could be isolated as white solid with a yield of $46 \%$ (over two steps).

${ }^{1} \mathrm{H}-\mathrm{NMR}\left(300 \mathrm{MHz}, \mathrm{CDCl}_{3}\right): \delta(\mathrm{ppm})=8.53(\mathrm{~s}, 1 \mathrm{H}), 8.10-7.97(\mathrm{~m}, 2 \mathrm{H}), 7.59(\mathrm{dt}, J=8.7,1.1 \mathrm{~Hz}, 2 \mathrm{H})$, $7.45(\mathrm{ddt}, J=8.5,6.5,1.2 \mathrm{~Hz}, 2 \mathrm{H}), 7.35(\mathrm{ddt}, J=9.9,6.6,1.6 \mathrm{~Hz}, 2 \mathrm{H}), 7.19(\mathrm{~s}, 1 \mathrm{H}), 5.23(\mathrm{td}, J=6.6,1.9$ $\mathrm{Hz}, 1 \mathrm{H}), 3.60(\mathrm{~s}, 1 \mathrm{H}), 2.99(\mathrm{ddt}, J=13.3,8.8,4.4 \mathrm{~Hz}, 1 \mathrm{H}), 2.79$ (ddd, $J=16.2,8.5,6.0 \mathrm{~Hz}, 1 \mathrm{H}), 2.48(\mathrm{tt}$, $J=8.0,4.2 \mathrm{~Hz}, 1 \mathrm{H}), 2.36(\mathrm{~s}, 3 \mathrm{H}), 2.01-1.90(\mathrm{~m}, 1 \mathrm{H}) .{ }^{13} \mathrm{C}-\mathrm{NMR}\left(75 \mathrm{MHz}, \mathrm{CDCl}_{3}\right) \delta(\mathrm{ppm})=164.5,156.6$, $144.4,135.1,134.4,131.4,131.4,130.3,130.2$, 128.5, 128.4, 127.4, 126.8, 126.3, 126.2, 125.7, $125.7,125.1,125.1,75.0,32.1,26.0,18.7$. 


\section{SI: Application of Crabtree/Pfaltz-type iridium complexes for the catalyzed asymmetric hydrogenation of an agrochemical building block}

2.1.3.11. (S)-4-methyl-2-(naphthalene-1-yl)-6,7-dihydro-5H-cyclopenta[b]pyridin-7ol (4k)<smiles>Cc1cc(-c2cccc3ccccc23)nc2c1CCC2O</smiles>

The reaction was performed once in our laboratory ( $2 \mathrm{mmol}$ scale) according to the above described procedure (1.1.3.). The product could be isolated as white solid with a yield of $96 \%$.

${ }^{1} \mathrm{H}-\mathrm{NMR}\left(300 \mathrm{MHz}, \mathrm{CDCl}_{3}\right): \delta(\mathrm{ppm})=8.04-7.97(\mathrm{~m}, 1 \mathrm{H}), 7.93-7.87(\mathrm{~m}, 2 \mathrm{H}), 7.57-7.40(\mathrm{~m}, 4 \mathrm{H})$, $7.25-7.22(\mathrm{~m}, 1 \mathrm{H}), 5.30-5.20(\mathrm{~m}, 1 \mathrm{H}), 3.51(\mathrm{br} \mathrm{s}, 1 \mathrm{H}), 3.06-2.91(\mathrm{~m}, 1 \mathrm{H}), 2.84-2.70(\mathrm{~m}, 1 \mathrm{H}), 2.61$ $-2.45(\mathrm{~m}, 1 \mathrm{H}), 2.38-2.30(\mathrm{~m}, 3 \mathrm{H}), 2.10-1.93(\mathrm{~m}, 1 \mathrm{H}) .{ }^{13} \mathrm{C}-\mathrm{NMR}(75 \mathrm{MHz}, \mathrm{CDCl}) \delta(\mathrm{ppm})=164.0$, $158.0,144.5,138.6,134.3,134.0,131.5,128.7,128.4,127.5,126.4,125.9,125.9,125.4,125.4,75.2$, $32.4,26.1,18.8$.

\subsubsection{2. (S)-2-(3,5-dimethylphenyl)-4-methyl-6,7-dihydro-5H-} cyclopenta[b]pyridin-7-ol (4l)

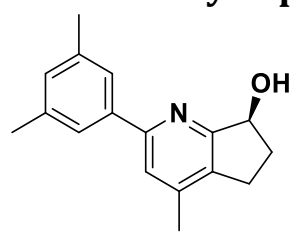

The reaction was performed once in our laboratory $(0.97 \mathrm{mmol}$ scale) according to the above described procedure (1.1.3.). The product could be isolated as white solid with a yield of $49 \%$.

${ }^{1} \mathrm{H}-\mathrm{NMR}\left(300 \mathrm{MHz}, \mathrm{CDCl}_{3}\right): \delta(\mathrm{ppm})=7.56(\mathrm{~s}, 2 \mathrm{H}), 7.39(\mathrm{~s}, 1 \mathrm{H}), 7.03(\mathrm{~s}, 1 \mathrm{H}), 5.26(\mathrm{dd}, J=7.4,5.8 \mathrm{~Hz}$, 1H), $3.22(\mathrm{~s}, 1 \mathrm{H}$ ), 3.00 (ddd, $J=16.3,9.0,4.0 \mathrm{~Hz}, 1 \mathrm{H}), 2.84-2.70(\mathrm{~m}, 1 \mathrm{H}), 2.58$ (dddd, $J=13.4,8.4$, 7.4, $4.0 \mathrm{~Hz}, 1 \mathrm{H}), 2.38$ (s, 6H), $2.34(\mathrm{~s}, 3 \mathrm{H}), 2.06$ (dddd, $J=13.4,9.0,6.8,5.8 \mathrm{~Hz}, 1 \mathrm{H}) .{ }^{13} \mathrm{C}-\mathrm{NMR}(75 \mathrm{MHz}$, $\left.\mathrm{CDCl}_{3}\right) \delta(\mathrm{ppm})=163.7,157.0,144.8,139.2,138.2,134.1,130.39,124.9,121.3,75.1,32.4,25.9,21.4$, 18.8 .

\subsubsection{3. (S)-2-(2,6-dimethylphenyl)-4-methyl-6,7-dihydro-5H-} cyclopenta[b]pyridin-7-ol (4m)

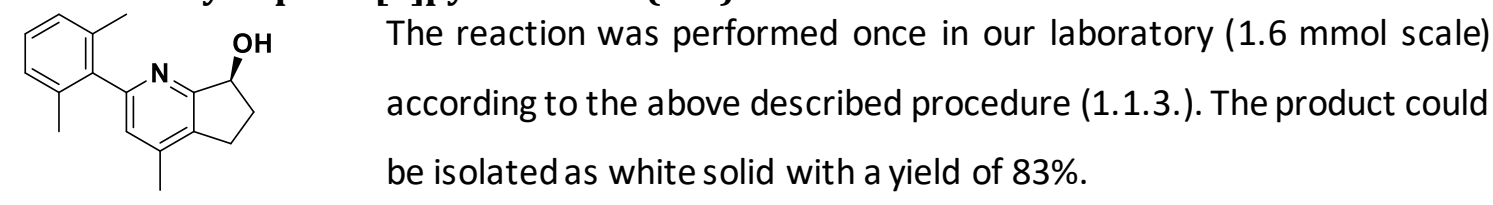

${ }^{1} \mathrm{H}-\mathrm{NMR}\left(300 \mathrm{MHz}, \mathrm{CDCl}_{3}\right): \delta(\mathrm{ppm})=7.23-7.12(\mathrm{~m}, 1 \mathrm{H}), 7.13-7.00(\mathrm{~m}, 2 \mathrm{H}), 6.90(\mathrm{~s}, 1 \mathrm{H}), 5.18(\mathrm{dd}$, $J=7.5,5.9 \mathrm{~Hz}, 1 \mathrm{H}$ ), $3.60(\mathrm{~s}, 1 \mathrm{H}$ ), 2.97 (ddd, $J=16.2,9.1,4.0 \mathrm{~Hz}, 1 \mathrm{H}$ ), $2.85-2.64(\mathrm{~m}, 1 \mathrm{H}), 2.51$ (dddd, $J=13.5,8.4,7.5,4.0 \mathrm{~Hz}, 1 \mathrm{H}), 2.31(\mathrm{~s}, 3 \mathrm{H}), 2.09-1.86(\mathrm{~m}, 7 \mathrm{H}) .{ }^{13} \mathrm{C}-\mathrm{NMR}\left(75 \mathrm{MHz}, \mathrm{CDCl}_{3}\right) \delta(\mathrm{ppm})=$ $164.1,158.4,144.4,140.3,136.0,133.6,127.8,127.5,124.4,75.0,32.1,25.9,20.3,18.7$.

2.1.3.14. (S)-2-mesityl-4-methyl-6,7-dihydro-5H-cyclopenta[b]pyridin-7-ol (4n)

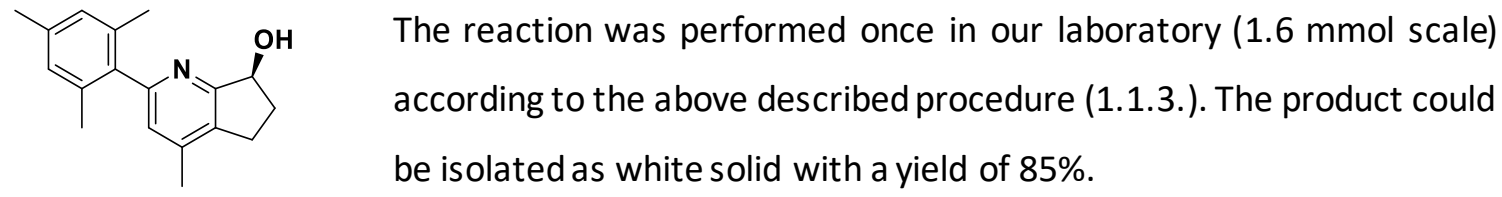


SI: Application of Crabtree/Pfaltz-type iridium complexes for the catalyzed asymmetric hydrogenation of an agrochemical building block

${ }^{1} \mathrm{H}-\mathrm{NMR}\left(300 \mathrm{MHz}, \mathrm{CDCl}_{3}\right): \delta(\mathrm{ppm})=6.91(\mathrm{~s}, 2 \mathrm{H}), 6.89(\mathrm{~s}, 1 \mathrm{H}), 5.19(\mathrm{dd}, J=7.5,5.9 \mathrm{~Hz}, 1 \mathrm{H}), 3.52(\mathrm{~s}$, 1H), 2.98 (ddd, $J=16.2,9.1,3.9 \mathrm{~Hz}, 1 \mathrm{H}$ ), $2.85-2.65(\mathrm{~m}, 1 \mathrm{H}$ ), 2.53 (dddd, $J=13.4,8.4,7.5,3.9 \mathrm{~Hz}$, 1H), 2.36-2.25 (m, 6H), 2.09-1.86 (m, 7H). ${ }^{13} \mathrm{C}-\mathrm{NMR}\left(75 \mathrm{MHz}, \mathrm{CDCl}_{3}\right) \delta(\mathrm{ppm})=163.9,158.6,144.3$, $137.5,137.3,135.8,133.4,128.2,124.6,75.0,32.1,25.9,21.1,20.2,18.7$.

\subsubsection{5. (R)-2-methyl-6,7-dihydro-5H-cyclopenta[b]pyridin-7-ol (4o)}<smiles>Cc1ccc2c(n1)C(O)CC2</smiles>

The reaction was performed once in our laboratory $(1.9 \mathrm{mmol}$ scale) according to the above described procedure (1.1.3.). The product could be isolated as white solid with a yield of $93 \%$.

${ }^{1} \mathrm{H}-\mathrm{NMR}\left(300 \mathrm{MHz}, \mathrm{CDCl}_{3}\right): \delta(\mathrm{ppm})=7.44(\mathrm{~d}, J=7.8 \mathrm{~Hz}, 1 \mathrm{H}), 6.98(\mathrm{~d}, J=7.8 \mathrm{~Hz}, 1 \mathrm{H}), 5.21$ (dd, $J=$ 7.4, $6.0 \mathrm{~Hz}, 1 \mathrm{H}$ ), $4.83(\mathrm{~s}, 1 \mathrm{H}$ ), 2.99 (dddt, $J=15.0,9.0,4.3,0.9 \mathrm{~Hz}, 1 \mathrm{H}$ ), 2.75 (dddt, $J=16.2,8.2,7.1$, $1.0 \mathrm{~Hz}, 1 \mathrm{H}), 2.56-2.38(\mathrm{~m}, 4 \mathrm{H}), 2.05$ (dddd, $J=13.2,8.9,7.0,5.9 \mathrm{~Hz}, 1 \mathrm{H}) .{ }^{13} \mathrm{C}-\mathrm{NMR}(75 \mathrm{MHz}, \mathrm{CDCl})$ $\delta(\mathrm{ppm})=164.4,156.8,133.6,133.3,122.4,74.3,33.1,27.2,23.6$.

2.1.3.16. (S)-2-(3,5-di-tert-butyl-4-methoxyphenyl)-4-methyl-6,7-dihydro-5Hcyclopenta[b]pyridin-7-ol (4p)

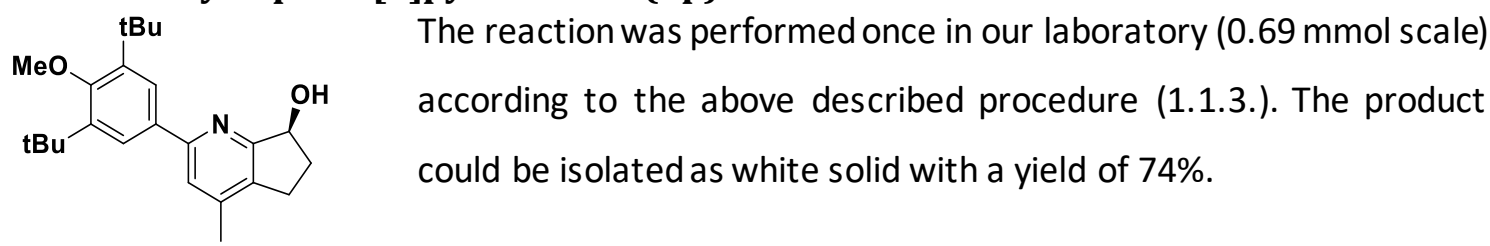

${ }^{1} \mathrm{H}-\mathrm{NMR}\left(300 \mathrm{MHz}, \mathrm{CDCl}_{3}\right): \delta(\mathrm{ppm})=7.75(\mathrm{~s}, 2 \mathrm{H}), 7.30(\mathrm{~s}, 1 \mathrm{H}), 5.27(\mathrm{dd}, J=7.4,5.4 \mathrm{~Hz}, 1 \mathrm{H}), 4.39(\mathrm{~s}$, 1H), $3.71(\mathrm{~s}, 3 \mathrm{H}$ ), 2.98 (ddd, $J=16.2,8.9,4.4 \mathrm{~Hz}, 1 \mathrm{H}$ ), $2.82-2.67(\mathrm{~m}, 1 \mathrm{H}), 2.51$ (dddd, $J=13.0,8.2$, $7.3,4.5 \mathrm{~Hz}, 1 \mathrm{H}$ ), 2.34 (d, $J=0.8 \mathrm{~Hz}, 3 \mathrm{H}$ ), 2.05 (dddd, $J=13.4,8.9,6.4,5.4 \mathrm{~Hz}, 1 \mathrm{H}$ ), $1.45(\mathrm{~s}, 18 \mathrm{H}) .{ }^{13} \mathrm{C}-$ $\operatorname{NMR}\left(75 \mathrm{MHz} \mathrm{CDCl}_{3}\right) \delta(\mathrm{ppm})=164.1,160.3,157.8,144.4,143.7,134.0,133.6,125.7,121.2,74.94$, $64.3,35.9,32.3,32.1,25.9,18.9$.

\subsubsection{7. (S)-4-methyl-2-(2,4,6-triisopropylphenyl)-6,7-dihydro-5H-} cyclopenta[b]pyridin-7-ol (4q)<smiles>Cc1cc(-c2c(C(F)(F)F)cc(C(C)C)cc2C(F)(F)F)nc2c1CCC2O</smiles>

The reaction was performed once in our laboratory $(0.66 \mathrm{mmol}$ scale) according to the above described procedure (1.1.3.). The product could be isolated as white solid with a yield of $88 \%$.

${ }^{1} \mathrm{H}-\mathrm{NMR}\left(300 \mathrm{MHz}, \mathrm{CDCl}_{3}\right): \delta(\mathrm{ppm})=7.06(\mathrm{~s}, 2 \mathrm{H}), 6.97(\mathrm{~s}, 1 \mathrm{H}), 5.17(\mathrm{dd}, J=7.6,5.7 \mathrm{~Hz}, 1 \mathrm{H}), 3.50(\mathrm{~s}$, $1 \mathrm{H}), 3.10-2.84(\mathrm{~m}, 2 \mathrm{H}), 2.77$ (ddd, $J=16.0,8.6,6.7 \mathrm{~Hz}, 1 \mathrm{H}), 2.60-2.37(\mathrm{~m}, 3 \mathrm{H}), 2.31(\mathrm{~s}, 3 \mathrm{H}), 2.11$ $-1.88(\mathrm{~m}, 1 \mathrm{H}), 1.29(\mathrm{dd}, J=6.9,0.7 \mathrm{~Hz}, 6 \mathrm{H}), 1.20-0.90(\mathrm{~m}, 12 \mathrm{H}) .{ }^{13} \mathrm{C}-\mathrm{NMR}\left(75 \mathrm{MHz}, \mathrm{CDCl}_{3}\right) \delta(\mathrm{ppm})$ $=163.7,158.8$, 148.7, 146.5, 146.3, 143.6, 136.2, 133.3, 125.0, 120.7, 120.6, 75.0, 34.5, 31.9, 30.4, $30.3,26.0,24.5,24.4,24.2,24.1,24.1,23.9,18.7$. 


\section{SI: Application of Crabtree/Pfaltz-type iridium complexes for the catalyzed asymmetric hydrogenation of an agrochemical building block}

\subsubsection{8. (S)-2-(4-(tert-butyl)-2,6-dimethylphenyl)-4-methyl-6,7-dihydro-5H- cyclopenta[b]pyridin-7-ol (4r)}

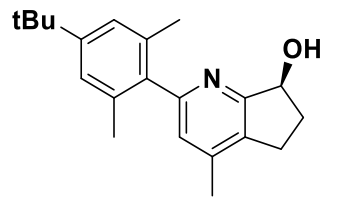

The reaction was performed once in our laboratory $(1.7 \mathrm{mmol}$ scale) according to the above described procedure (1.1.3.) using TBAF $(4.0$ equiv). The product could be isolated as white solid with a yield of $82 \%$.

${ }^{1} \mathrm{H}-\mathrm{NMR}\left(400 \mathrm{MHz}, \mathrm{CDCl}_{3}\right): \delta(\mathrm{ppm})=7.11(\mathrm{~s}, 2 \mathrm{H}), 6.94(\mathrm{~s}, 1 \mathrm{H}), 5.20(\mathrm{dd}, J=7.5,5.9 \mathrm{~Hz}, 1 \mathrm{H}), 3.26$ (s, 1H), 2.99 (ddd, $J=16.3,9.1,3.8 \mathrm{~Hz}, 1 \mathrm{H}$ ), $2.84-2.68(\mathrm{~m}, 1 \mathrm{H}$ ), 2.55 (dddd, $J=13.4,8.4,7.5,3.8$ $\mathrm{Hz}, 1 \mathrm{H}), 2.31(\mathrm{~s}, 3 \mathrm{H}), 2.10-1.91(\mathrm{~m}, 7 \mathrm{H}), 1.33(\mathrm{~s}, 9 \mathrm{H}) .{ }^{13} \mathrm{C}-\mathrm{NMR}\left(100 \mathrm{MHz}, \mathrm{CDCl}_{3}\right) \delta(\mathrm{ppm})=163.8$, 158.6, 150.6, 144.4, 137.3, 135.4, 133.4, 124.6 (2xC), 75.1, 34.4, 32.0, 31.4, 26.0, 20.6, 18.7.

\subsubsection{Synthesis of Iridium complexes}
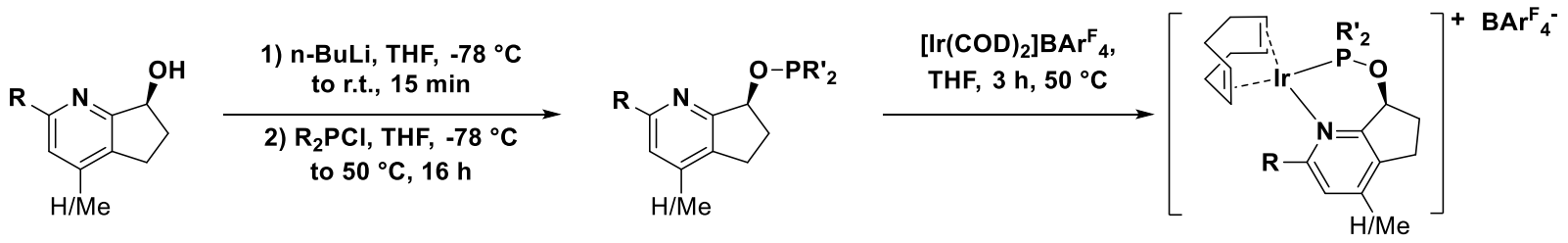

Literature: First step analogue to L. Wang, H. Neumann, A. Spannenberg, M. Beller, Chem. Commun. 2017, 53, 7469. Second step analogue to D. H. Woodmansee, M.-A. Müller, M. Neuburger, A. Pfaltz Chem. Sci. , 2010, 1, 72.

Procedure of ligand synthesis (under Ar): A solution of alcohol precursor in THF $(0.25 \mathrm{mmol}$, in $5.0 \mathrm{~mL}$ THF) was cooled to $-78^{\circ} \mathrm{C}$ and $\mathrm{n}$-BuLi ( $0.1 \mathrm{~mL}$ of a $2.5 \mathrm{M}$ n-BuLi solution in hexane; $0.25 \mathrm{mmol}$; 1 eq.) was added dropwise to the continuously stirred solution. After completion of the addition the solution was allowed to warm to room temperature and was stirred at this temperature for further $30 \mathrm{~min}$. The solution was cooled to $-78^{\circ} \mathrm{C}$ again and $\mathrm{R}_{2} \mathrm{PCl}(0.25 \mathrm{mmol}, 1$ eq. $)$ was added to the continuously stirred solution. The mixture was allowed to warm to room temperature and subsequently heated to $50{ }^{\circ} \mathrm{C}$ and kept at this temperature overnight. The theoretical yield of ligand was calculated using ${ }^{31} \mathrm{P}-\mathrm{NMR}$ and the ligand was used for the next step without further purification.

Procedure of complexation (under $\mathrm{Ar}$ ): To the crude ligand solution was added $\left[\operatorname{Ir}(\mathrm{COD})_{2}\right] \mathrm{BAr}_{4}$ (as a solid, 1eq. based on the theoretical yield). The resulting mixture was heated to $50{ }^{\circ} \mathrm{C}$ and kept at this temperature for $3 \mathrm{~h}$.

Work-up (under air): After cooling to room temperature all volatiles are removed under reduced pressure. The residue is re-dissolved in DCM, loaded onto silica and subsequently purified by column chromatography on silica (using either pentane/diethyl ether or pentane/MTBE as eluent). Using one of these solvent mixtures a yellowish side product was eluted first. Afterwards the solvent system was switched to pure DCM to give the desired complex. 
SI: Application of Crabtree/Pfaltz-type iridium complexes for the catalyzed asymmetric hydrogenation of an agrochemical building block

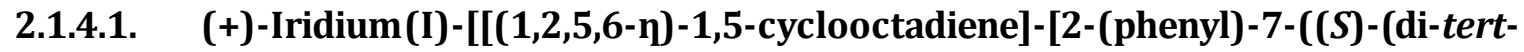
butylphosphinite-kP)-6,7-dihydro-5H-

cyclopenta[b]pyridine-kN]-tetrakis(3,5-

bis(trifluoromethyl)phenyl)borate(-) (5a)

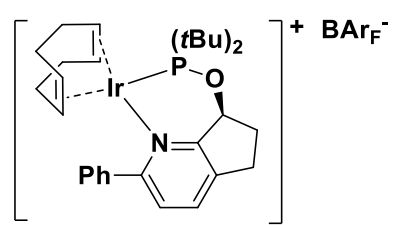

Complex 5a was obtained from Bayer AG and can be seen as the benchmark catalyst for the aimed reaction.

${ }^{1} \mathrm{H}-\mathrm{NMR}\left(300 \mathrm{MHz}, \mathrm{CDCl}_{3}\right): \delta(\mathrm{ppm})=8.27-8.19(\mathrm{~m}, 2 \mathrm{H}), 7.81-7.59(\mathrm{~m}, 13 \mathrm{H}), 7.51(\mathrm{~s}, 4 \mathrm{H}), 5.72$ (ddd, $J=8.2,5.6,4.1 \mathrm{~Hz}, 1 \mathrm{H}$ ), $5.31(\mathrm{t}, J=6.9 \mathrm{~Hz}, 1 \mathrm{H}$ ), $4.47(\mathrm{~s}, 1 \mathrm{H}$ ), $4.12(\mathrm{~d}, J=6.5 \mathrm{~Hz}, 1 \mathrm{H}$ ), 3.10 (ddd, $J=17.1,9.6,4.3 \mathrm{~Hz}, 1 \mathrm{H}), 3.01-2.85(\mathrm{~m}, 1 \mathrm{H}), 2.85-2.69(\mathrm{~m}, 1 \mathrm{H}), 2.52(\mathrm{p}, J=7.7 \mathrm{~Hz}, 1 \mathrm{H}), 2.44-1.99$ (m, 4H), 1.86 (dd, $J=15.3,8.3 \mathrm{~Hz}, 1 \mathrm{H}$ ), 1.65 (dd, $J=14.8,8.6 \mathrm{~Hz}, 2 \mathrm{H}), 1.49$ (d, $J=13.7 \mathrm{~Hz}, 9 \mathrm{H}$ ), $1.32-$ $1.20(\mathrm{~m}, 2 \mathrm{H}), 1.04(\mathrm{~d}, J=14.5 \mathrm{~Hz}, 9 \mathrm{H}) .{ }^{31}$ P-NMR $\left(122 \mathrm{MHz}, \mathrm{CDCl}_{3}\right) \delta=142.1$. HR-MS (ESI) $\mathrm{m} / z$ calcd for $\mathrm{C}_{30} \mathrm{H}_{42} \mathrm{NOPIr}[\mathrm{M}]^{+} 656.2633$ found 656.2634 .

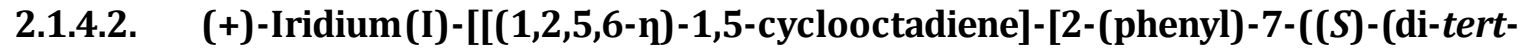
butylphosphinite-kP)-4-methyl-6,7-dihydro-5Hcyclopenta[b]pyridine-kN]-tetrakis(3,5bis(trifluoromethyl)phenyl)borate(-) (5b)

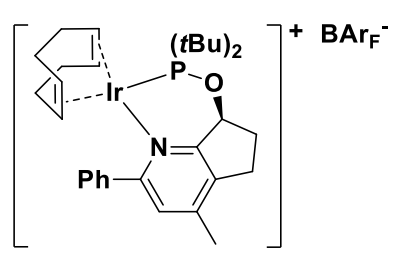

The reaction was performed once in our laboratory according to the above described procedure (1.1.4.), using $\mathbf{4 b}$ as starting material. The theoretical yield of the ligand was $44 \%$. The complex could be isolated as an orange solid $\left(89.5 \mathrm{mg} ; 53 \%\right.$ based on $\left.\left[\operatorname{Ir}(\mathrm{COD})_{2}\right] \mathrm{BAr}_{4}{ }_{4}\right)$.

${ }^{1} \mathrm{H}-\mathrm{NMR}\left(300 \mathrm{MHz}, \mathrm{CD}_{2} \mathrm{Cl}_{2}\right): \delta(\mathrm{ppm})=8.26(\mathrm{dd}, J=7.9,1.7 \mathrm{~Hz}, 2 \mathrm{H}), 7.81-7.36(\mathrm{~m}, 16 \mathrm{H}), 5.75(\mathrm{dt}, J$ $=8.0,5.2 \mathrm{~Hz}, 1 \mathrm{H}), 5.34-5.29(\mathrm{~m}, 1 \mathrm{H}), 4.51(\mathrm{q}, J=5.3,3.2 \mathrm{~Hz}, 1 \mathrm{H}), 4.11(\mathrm{dq}, J=12.5,7.6,5.9 \mathrm{~Hz}, 1 \mathrm{H})$, $3.08(\mathrm{ddd}, J=16.6,10.3,3.8 \mathrm{~Hz}, 1 \mathrm{H}), 2.99-2.70(\mathrm{~m}, 2 \mathrm{H}), 2.61-2.00(\mathrm{~m}, 8 \mathrm{H}), 1.92-1.79(\mathrm{~m}, 1 \mathrm{H})$, $1.69(\mathrm{dd}, J=14.8,8.1 \mathrm{~Hz}, 1 \mathrm{H}), 1.51(\mathrm{~s}, 9 \mathrm{H}), 1.29-1.24(\mathrm{~m}, 3 \mathrm{H}), 1.06(\mathrm{~d}, J=14.4 \mathrm{~Hz}, 9 \mathrm{H}) .{ }^{31}$ P-NMR (122 $\left.\mathrm{MHz}, \mathrm{CD}_{2} \mathrm{Cl}_{2}\right) \delta(\mathrm{ppm})=$ 142.1. ${ }^{19} \mathrm{~F}-\mathrm{NMR}\left(282 \mathrm{MHz}, \mathrm{CD}_{2} \mathrm{Cl}_{2}\right) \delta(\mathrm{ppm})=-62.9$. HR-MS (ESI) $\mathrm{m} / z$ calcd for $\mathrm{C}_{31} \mathrm{H}_{44} \mathrm{NOPIr}[\mathrm{M}]^{+} 670.2790$ found 670.2798 . 
SI: Application of Crabtree/Pfaltz-type iridium complexes for the catalyzed asymmetric hydrogenation of an agrochemical building block

2.1.4.3. (+)-Iridium(I)-[[(1,2,5,6-n)-1,5-cyclooctadiene]-[2-(4-methoxy-phenyl)-7((S)-(di-tert-butylphosphinite-kP)-4-methyl-6,7-dihydro-5Hcyclopenta[b]pyridine-kN]-tetrakis(3,5bis(trifluoromethyl)phenyl)borate(-) (5c)

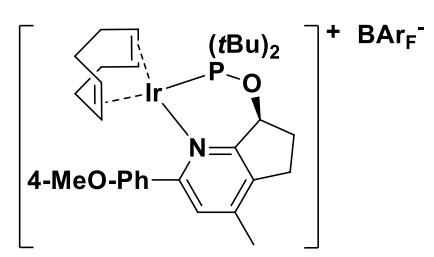

The reaction was performed once in our laboratory according to the above described procedure (1.1.4.), using $\mathbf{4 g}$ as starting material. The theoretical yield of the ligand was $51 \%$. The complex could be isolated as an orange solid $\left(78.0 \mathrm{mg} ; 39 \%\right.$ based on $\left.\left[\operatorname{Ir}(\mathrm{COD})_{2}\right] \mathrm{BAr}_{4}{ }_{4}\right)$.

${ }^{1} \mathrm{H}-\mathrm{NMR}\left(300 \mathrm{MHz}, \mathrm{CDCl}_{3}\right): \delta(\mathrm{ppm})=8.22(\mathrm{~d}, J=8.7 \mathrm{~Hz}, 2 \mathrm{H}), 7.80-7.63(\mathrm{~m}, 8 \mathrm{H}), 7.63-7.43(\mathrm{~m}, 5 \mathrm{H})$, $7.16(\mathrm{~d}, J=8.8 \mathrm{~Hz}, 2 \mathrm{H}), 5.82-5.66(\mathrm{~m}, 1 \mathrm{H}), 5.37-5.22(\mathrm{~m}, 1 \mathrm{H}), 4.56-4.41(\mathrm{~m}, 1 \mathrm{H}), 4.18-4.00(\mathrm{~m}$, $1 \mathrm{H}), 3.93(\mathrm{~s}, 3 \mathrm{H}), 3.12-2.97(\mathrm{~m}, 1 \mathrm{H}), 2.96-2.74(\mathrm{~m}, 2 \mathrm{H}), 2.70-2.56(\mathrm{~m}, 1 \mathrm{H}), 2.43(\mathrm{~s}, 3 \mathrm{H}), 2.41-$ $2.03(\mathrm{~m}, 4 \mathrm{H}), 1.96-1.84(\mathrm{~m}, 1 \mathrm{H}), 1.72(\mathrm{dd}, J=14.6,7.9 \mathrm{~Hz}, 1 \mathrm{H}), 1.51(\mathrm{~d}, J=15.0 \mathrm{~Hz}, 9 \mathrm{H}), 1.34-1.23$ $(\mathrm{m}, 3 \mathrm{H}), 1.05(\mathrm{~d}, J=14.4 \mathrm{~Hz}, 9 \mathrm{H}) .{ }^{31} \mathrm{P}-\mathrm{NMR}\left(122 \mathrm{MHz}, \mathrm{CD}_{2} \mathrm{Cl}_{2}\right) \delta(\mathrm{ppm})=141.9 .{ }^{19} \mathrm{~F}-\mathrm{NMR}(282 \mathrm{MHz}$, $\left.\mathrm{CD}_{2} \mathrm{Cl}_{2}\right) \delta(\mathrm{ppm})=-62.9$. HR-MS $(\mathrm{ESI}) \mathrm{m} / z$ calcd for $\mathrm{C}_{32} \mathrm{H}_{46} \mathrm{NO}_{2} \mathrm{PIr}[\mathrm{M}]^{+} 700.2895$ found 700.2899.

2.1.4.4. (+)-Iridium(I)-[[(1,2,5,6-n)-1,5-cyclooctadiene]-[2-(phenyl)-7-((R)(di-cyclohexylphosphinite-kP)-6,7-dihydro-5Hcyclopenta[b]pyridine-kN]-tetrakis(3,5bis(trifluoromethyl)phenyl)borate(-) (5d)

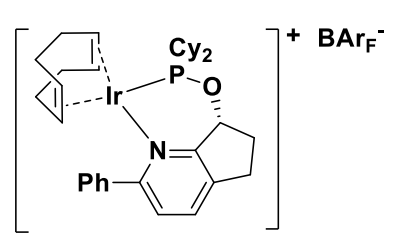

The reaction was performed once in our laboratory according to the above described procedure (1.1.4.), using $4 \mathrm{a}$ as starting material. The theoretical yield of the ligand was $93 \%$. The complex could be isolated as an orange solid (197 $\mathrm{mg} ; 54 \%$ based on $\left.\left[\operatorname{Ir}(\mathrm{COD})_{2}\right] \mathrm{BAr}_{4}{ }_{4}\right)$.

${ }^{1} \mathrm{H}-\mathrm{NMR}(300 \mathrm{MHz}, \mathrm{CDCl})$ ): $\delta(\mathrm{ppm})=8.31-8.22(\mathrm{~m}, 2 \mathrm{H}), 7.81-7.59(\mathrm{~m}, 13 \mathrm{H}), 7.51(\mathrm{~s}, 4 \mathrm{H}), 5.73(\mathrm{dt}$, $J=9.1,4.8 \mathrm{~Hz}, 1 \mathrm{H}), 4.79(\mathrm{~s}, 1 \mathrm{H}), 4.58(\mathrm{~s}, 1 \mathrm{H}), 3.50(\mathrm{~s}, 1 \mathrm{H}), 3.15-3.00(\mathrm{~m}, 1 \mathrm{H}), 2.99-2.83(\mathrm{~m}, 1 \mathrm{H})$, $2.83-2.55(\mathrm{~m}, 2 \mathrm{H}), 2.49-1.73(\mathrm{~m}, 15 \mathrm{H}), 1.42-0.92(\mathrm{~m}, 13 \mathrm{H}), 0.54(\mathrm{~s}, 1 \mathrm{H}) .{ }^{31} \mathrm{P}-\mathrm{NMR}(122 \mathrm{MHz}$, $\left.\mathrm{CDCl}_{3}\right) \delta=121.3 .{ }^{19} \mathrm{~F}-\mathrm{NMR}\left(282 \mathrm{MHz}, \mathrm{CDCl}_{3}\right) \delta=-62.4$. HR-MS (ESI) $\mathrm{m} / \mathrm{z}$ calcd for $\mathrm{C}_{34} \mathrm{H}_{46} \mathrm{NOPIr}[\mathrm{M}]^{+}$ 708.2947 found 700.2947 .

2.1.4.5. (+)-Iridium(I)-[[(1,2,5,6 ฤ)-1,5-cyclooctadiene]-[2-(phenyl)-7-((R)-(di ethylphosphinite-kP)-6,7-dihydro-5H-cyclopenta[b]pyridine-kN] tetrakis(3,5-bis(trifluoromethyl)phenyl)borate(-) (5e)

The reaction was performed once in our laboratory according to the

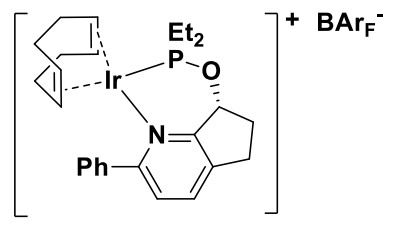
above described procedure (1.1.4.), using $4 a$ as starting material. The theoretical yield of the ligand was $94 \%$. The complex could be isolated as an orange solid (134 mg; 95\% purity based on ${ }^{31} \mathrm{P}-\mathrm{NMR}$; $39 \%$ based on $\left.\left[\operatorname{Ir}(\mathrm{COD})_{2}\right] \mathrm{BAr}_{4}\right)$. 
SI: Application of Crabtree/Pfaltz-type iridium complexes for the catalyzed asymmetric hydrogenation of an agrochemical building block

${ }^{1} \mathrm{H}-\mathrm{NMR}\left(300 \mathrm{MHz}, \mathrm{CDCl}_{3}\right): \delta(\mathrm{ppm})=8.00-7.92(\mathrm{~m}, 2 \mathrm{H}), 7.81-7.76(\mathrm{~m}, 1 \mathrm{H}), 7.75-7.64(\mathrm{~m}, 10 \mathrm{H})$, $7.62-7.55(\mathrm{~m}, 2 \mathrm{H}), 7.52(\mathrm{~d}, \mathrm{~J}=1.9 \mathrm{~Hz}, 4 \mathrm{H}), 5.88(\mathrm{dt}, \mathrm{J}=8.3,4.9 \mathrm{~Hz}, 1 \mathrm{H}), 4.52(\mathrm{dt}, \mathrm{J}=8.3,4.2 \mathrm{~Hz}, 1 \mathrm{H})$, $4.37(\mathrm{ddt}, \mathrm{J}=7.4,5.0,2.5 \mathrm{~Hz}, 1 \mathrm{H}), 3.61(\mathrm{td}, \mathrm{J}=8.0,3.8 \mathrm{~Hz}, 1 \mathrm{H}), 3.17-2.64(\mathrm{~m}, 4 \mathrm{H}), 2.34-1.79(\mathrm{~m}$, $9 \mathrm{H}), 1.68-1.55(\mathrm{~m}, 1 \mathrm{H}), 1.36-0.90(\mathrm{~m}, 9 \mathrm{H}) .{ }^{31} \mathrm{P}-\mathrm{NMR}\left(122 \mathrm{MHz}, \mathrm{CDCl}_{3}\right) \delta=116.4$ (mayor product; 95\%), 111.8 (minor species; 5\%). ${ }^{19}$ F-NMR (282 MHz, $\mathrm{CDCl}_{3}$ ) $\delta=-62.4$. HR-MS (ESI) $\mathrm{m} / \mathrm{z}$ calcd for $\mathrm{C}_{26} \mathrm{H}_{34} \mathrm{NOPIr}[\mathrm{M}]^{+} 600.2006$ found 600.2006.

2.1.4.6. (+)-Iridium(I)-[[(1,2,5,6-n)-1,5-cyclooctadiene]-[2-(phenyl)-7-((S)(di-cyclohexylphosphinite-kP)-4-methyl-6,7-dihydro-5Hcyclopenta[b]pyridine-kN]-tetrakis(3,5bis(trifluoromethyl)phenyl)borate(-) (5f)

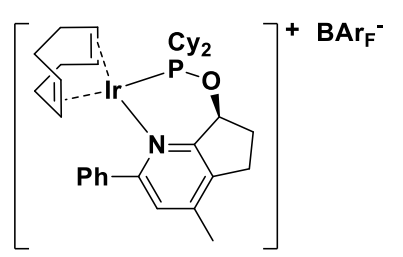

The reaction was performed once in our laboratory according to the above described procedure (1.1.4.), using $\mathbf{4 b}$ as starting material. The theoretical yield of the ligand was $93 \%$. The complex could be isolated as an orange solid $\left(241 \mathrm{mg} ; 71 \%\right.$ based on $\left.\left[\operatorname{Ir}(\mathrm{COD})_{2}\right] \mathrm{BAr}_{4}\right)$.

${ }^{1} \mathrm{H}-\mathrm{NMR}\left(300 \mathrm{MHz}, \mathrm{CD}_{2} \mathrm{Cl}_{2}\right): \delta(\mathrm{ppm})=8.38-8.14(\mathrm{~m}, 2 \mathrm{H}), 7.83-7.43(\mathrm{~m}, 16 \mathrm{H}), 5.76(\mathrm{dt}, \mathrm{J}=7.7,4.9$ $\mathrm{Hz}, 1 \mathrm{H}), 4.81(\mathrm{t}, J=7.6 \mathrm{~Hz}, 1 \mathrm{H}), 4.70-4.46(\mathrm{~m}, 1 \mathrm{H}), 3.56-3.39(\mathrm{~m}, 1 \mathrm{H}), 3.06(\mathrm{ddd}, J=16.7,10.3,3.6$ $H z, 1 H), 2.98-2.73(m, 2 H), 2.71-2.57(m, 1 H), 2.44(s, 3 H), 2.41-2.02(m, 6 H), 2.00-1.75(m$, 7H), $1.72-1.54(\mathrm{~m}, 4 \mathrm{H}), 1.46-0.94(\mathrm{~m}, 13 \mathrm{H}), 0.72-0.50(\mathrm{~m}, 1 \mathrm{H}) .{ }^{31} \mathrm{P}-\mathrm{NMR}\left(122 \mathrm{MHz}, \mathrm{CD}_{2} \mathrm{Cl} 2\right) \delta$ $(\mathrm{ppm})=121.3 .{ }^{19} \mathrm{~F}-\mathrm{NMR}\left(282 \mathrm{MHz}, \mathrm{CD}_{2} \mathrm{Cl} 2\right) \delta(\mathrm{ppm})=-62.9$. HR-MS $(\mathrm{ESI}) \mathrm{m} / \mathrm{z}$ calcd for $\mathrm{C}_{35} \mathrm{H}_{48} \mathrm{NOPIr}$ $[\mathrm{M}]^{+} 722.3103$ found 722.3116 .

2.1.4.7. (+)-Iridium(I)-[[(1,2,5,6-n)-1,5-cyclooctadiene]-[2-(phenyl)-7-((S)-(di-isopropylphosphinite-kP)-4-methyl-6,7-dihydro-5H-

cyclopenta[b]pyridine-kN]-tetrakis(3,5-

bis(trifluoromethyl)phenyl)borate(-) (5g)

The reaction was performed once in our laboratory $(0.5 \mathrm{mmol})$

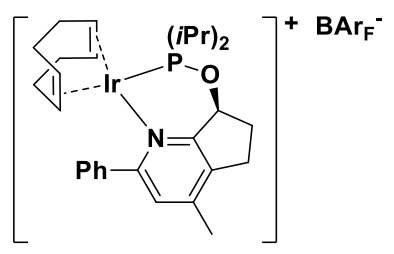

according to the above described procedure, but after the addition of $\mathrm{CIP}(i \mathrm{Pr})_{2}$ was completed, the reaction mixture was stirred at $\mathrm{RT}$ for $16 \mathrm{~h}$ (1.1.4.), using $\mathbf{4 b}$ as starting material. The theoretical yield of the ligand was $95 \%$. The complex could be isolated as an orange solid $(605 \mathrm{mg}$; $85 \%$ based on $\left.\left[\operatorname{Ir}(\mathrm{COD})_{2}\right] \mathrm{BAr}_{4}\right)$.

${ }^{1} \mathrm{H}-\mathrm{NMR}\left(300 \mathrm{MHz}, \mathrm{CDCl}_{3}\right): \delta(\mathrm{ppm})=8.17(\mathrm{dd}, J=7.1,1.8 \mathrm{~Hz}, 2 \mathrm{H}), 7.78-7.40(\mathrm{~m}, 16 \mathrm{H}), 5.74(\mathrm{dt}, J$ $=9.0,4.7 \mathrm{~Hz}, 1 \mathrm{H}), 4.83(\mathrm{t}, J=6.9 \mathrm{~Hz}, 1 \mathrm{H}), 4.61(\mathrm{dt}, J=8.7,4.1 \mathrm{~Hz}, 1 \mathrm{H}), 3.62-3.53(\mathrm{~m}, 1 \mathrm{H}), 3.11-2.94$ $(\mathrm{m}, 1 \mathrm{H}), 2.91-2.67(\mathrm{~m}, 2 \mathrm{H}), 2.67-2.44(\mathrm{~m}, 2 \mathrm{H}), 2.39(\mathrm{~s}, 3 \mathrm{H}), 2.36-1.93(\mathrm{~m}, 6 \mathrm{H}), 1.85(\mathrm{dd}, J=14.5$, $7.3 \mathrm{~Hz}, 1 \mathrm{H}$ ), $1.46(\mathrm{dd}, \mathrm{J}=15.2,7.1 \mathrm{~Hz}, 3 \mathrm{H}), 1.39-1.31(\mathrm{~m}, 1 \mathrm{H}), 1.23(\mathrm{dd}, J=13.3,6.9 \mathrm{~Hz}, 4 \mathrm{H}), 1.08$ (dd, $J=19.4,7.1 \mathrm{~Hz}, 3 \mathrm{H}), 0.52(\mathrm{dd}, J=15.5,7.1 \mathrm{~Hz}, 3 \mathrm{H}) .{ }^{31} \mathrm{P}-\mathrm{NMR}\left(122 \mathrm{MHz}, \mathrm{CDCl}_{3}\right) \delta(\mathrm{ppm})=129.5$. 
SI: Application of Crabtree/Pfaltz-type iridium complexes for the catalyzed asymmetric hydrogenation of an agrochemical building block

${ }^{19} \mathrm{~F}-\mathrm{NMR}\left(282 \mathrm{MHz}, \mathrm{CDCl}_{3}\right) \delta(\mathrm{ppm})=-62.4$. HR-MS $(\mathrm{ESI}) \mathrm{m} / \mathrm{z}$ calcd for $\mathrm{C}_{29} \mathrm{H}_{40} \mathrm{NOPIr}[\mathrm{M}]^{+} 642.2477$ found 642.2480 .

2.1.4.8. (+)-Iridium(I)-[[(1,2,5,6-n)-1,5-cyclooctadiene]-[2-(phenyl)-7-((S)(di-cyclopentylphosphinite-kP)-4-methyl-6,7-dihydro-5Hcyclopenta[b]pyridine- $\mathrm{kN}]-$ tetrakis(3,5-

bis(trifluoromethyl)phenyl)borate(-) (5h)

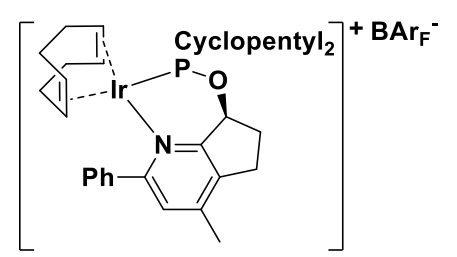

The reaction was performed once in our laboratory according to the above described procedure (1.1.4.), using $4 \mathbf{b}$ as starting material. The theoretical yield of the ligand was $90 \%$. The complex could be isolated as an orange solid (261 $\mathrm{mg} ; 75 \%$ based on $\left.\left[\operatorname{Ir}(\mathrm{COD})_{2}\right] \mathrm{BAr}_{4}{ }_{4}\right)$.

${ }^{1} \mathrm{H}-\mathrm{NMR}\left(300 \mathrm{MHz}, \mathrm{CD}_{2} \mathrm{Cl}_{2}\right): \delta(\mathrm{ppm})=8.28-8.11(\mathrm{~m}, 2 \mathrm{H}), 7.93-7.45(\mathrm{~m}, 16 \mathrm{H}), 5.81(\mathrm{dt}, J=9.3,5.0$ $\mathrm{Hz}, 1 \mathrm{H}), 4.89(\mathrm{t}, J=6.9 \mathrm{~Hz}, 1 \mathrm{H}), 4.72-4.51(\mathrm{~m}, 1 \mathrm{H}), 3.86-3.66(\mathrm{~m}, 1 \mathrm{H}), 3.18-3.04(\mathrm{~m}, 1 \mathrm{H}), 3.04-$ $2.57(\mathrm{~m}, 4 \mathrm{H}), 2.49(\mathrm{~s}, 3 \mathrm{H}), 2.46-1.61(\mathrm{~m}, 18 \mathrm{H}), 1.56-1.36(\mathrm{~m}, 5 \mathrm{H}), 1.36-1.14(\mathrm{~m}, 1 \mathrm{H}), 1.13-0.93$ $(\mathrm{m}, 1 \mathrm{H}), 0.77-0.66(\mathrm{~m}, 1 \mathrm{H}) .{ }^{31} \mathrm{P}-\mathrm{NMR}\left(122 \mathrm{MHz}, \mathrm{CD}_{2} \mathrm{Cl}_{2}\right) \delta(\mathrm{ppm})=129.4 .{ }^{19} \mathrm{~F}-\mathrm{NMR}\left(282 \mathrm{MHz}, \mathrm{CD}_{2} \mathrm{Cl}_{2}\right)$ $\delta(\mathrm{ppm})=-62.9$. HR-MS (ESI) $\mathrm{m} / z$ calcd for $\mathrm{C}_{33} \mathrm{H}_{44} \mathrm{NOPIr}[\mathrm{M}]^{+} 694.2790$ found 694.2789 .

2.1.4.9. (+)-Iridium(I)-[[(1,2,5,6-n)-1,5-cyclooctadiene]-[7-((R)(di-cyclopentylphosphinite-kP)-2-((4-tert-butyl)phenyl)-6,7-dihydro-5Hcyclopenta[b]pyridine-kN]-tetrakis(3,5-

bis(trifluoromethyl)phenyl)borate(-) (5i)

The reaction was performed once in our laboratory using

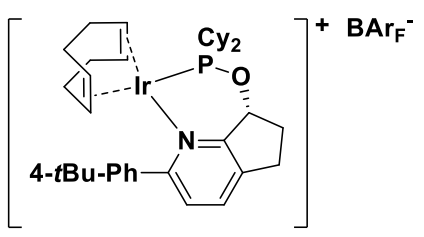

$\left[\operatorname{Ir}(\mathrm{COD})_{2}\right]_{\mathrm{BAr}_{4}}(287 \mathrm{mg}, 0.225 \mathrm{mmol})$ according to the above described procedure (1.1.4.), using $4 \mathrm{e}$ as starting material. The complex could be isolated as an orange solid ( $261 \mathrm{mg} ; 74 \%$ based on $\left.\left[\operatorname{Ir}(\mathrm{COD})_{2}\right] \mathrm{BAr}_{4}{ }_{4}\right)$.

${ }^{1} \mathrm{H}-\mathrm{NMR}\left(300 \mathrm{MHz}, \mathrm{CD}_{2} \mathrm{Cl}_{2}\right): \delta(\mathrm{ppm})=8.25(\mathrm{~d}, J=8.3 \mathrm{~Hz}, 2 \mathrm{H}), 7.87(\mathrm{~d}, J=8.1 \mathrm{~Hz}, 1 \mathrm{H}), 7.81-7.64(\mathrm{~m}$, $11 \mathrm{H}), 7.56(\mathrm{~s}, 4 \mathrm{H}), 5.74(\mathrm{dt}, J=8.2,4.6 \mathrm{~Hz}, 1 \mathrm{H}), 4.95-4.74(\mathrm{~m}, 1 \mathrm{H}), 4.74-4.51(\mathrm{~m}, 1 \mathrm{H}), 3.60-3.45$ $(\mathrm{m}, 1 \mathrm{H}), 3.23-2.91(\mathrm{~m}, 2 \mathrm{H}), 2.90-2.70(\mathrm{~m}, 1 \mathrm{H}), 2.67-2.50(\mathrm{~m}, 1 \mathrm{H}), 2.52-2.23(\mathrm{~m}, 4 \mathrm{H}), 2.28-2.04$ $(\mathrm{m}, 3 \mathrm{H}), 2.04-1.77(\mathrm{~m}, 7 \mathrm{H}), 1.69-1.58(\mathrm{~m}, 4 \mathrm{H}), 1.45-1.26(\mathrm{~m}, 17 \mathrm{H}), 1.17-0.95(\mathrm{~m}, 4 \mathrm{H}), 0.68-$ $0.42(\mathrm{~m}, 1 \mathrm{H}) .{ }^{31} \mathrm{P}-\mathrm{NMR}\left(122 \mathrm{MHz}, \mathrm{CD}_{2} \mathrm{Cl}_{2}\right) \delta(\mathrm{ppm})=121.1 .{ }^{19} \mathrm{~F}-\mathrm{NMR}\left(282 \mathrm{MHz}, \mathrm{CD}_{2} \mathrm{Cl}_{2}\right) \delta(\mathrm{ppm})=-$ 62.9. HR-MS (ESI) $\mathrm{m} / \mathrm{z}$ calcd for $\mathrm{C}_{38} \mathrm{H}_{54} \mathrm{NOPIr}[\mathrm{M}]^{+} 764.3572$ found 764.3586 . 
SI: Application of Crabtree/Pfaltz-type iridium complexes for the catalyzed asymmetric hydrogenation of an agrochemical building block

2.1.4.10. (+)-Iridium(I)-[[(1,2,5,6- $)-1,5$-cyclooctadiene]-[7-((S)(di-cyclohexylphosphinite-kP)-2-((4-trifluoromethyl)phenyl)-4-methyl6,7-dihydro-5H-cyclopenta[b]pyridine-kN]-tetrakis(3,5bis(trifluoromethyl)phenyl)borate(-) (5j)

The reaction was performed once in our laboratory using

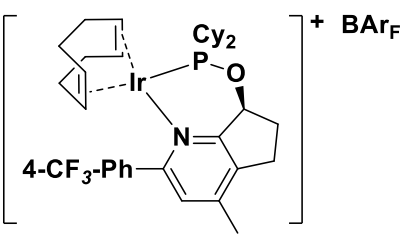
$\left[\operatorname{Ir}(\mathrm{COD})_{2}\right]_{\mathrm{BAr}_{4}}(287 \mathrm{mg}, 0.225 \mathrm{mmol})$ according to the above described procedure (1.1.4.), using $\mathbf{4 h}$ as starting material. The complex could be isolated as an orange solid $(180.0 \mathrm{mg} ; 48 \%$ based on $\left.\left[\operatorname{lr}(\mathrm{COD})_{2}\right] \mathrm{BAr}_{4}\right)$.

${ }^{1} \mathrm{H}-\mathrm{NMR}\left(300 \mathrm{MHz}, \mathrm{CD}_{2} \mathrm{Cl}_{2}\right): \delta(\mathrm{ppm})=8.46(\mathrm{~d}, J=7.9 \mathrm{~Hz}, 2 \mathrm{H}), 7.94(\mathrm{~d}, J=8.0 \mathrm{~Hz}, 2 \mathrm{H}), 7.82-7.38(\mathrm{~m}$, $13 \mathrm{H}), 5.83-5.69(\mathrm{~m}, 1 \mathrm{H}), 4.94-4.78(\mathrm{~m}, 1 \mathrm{H}), 4.73-4.54(\mathrm{~m}, 1 \mathrm{H}), 3.65-3.38(\mathrm{~m}, 1 \mathrm{H}), 3.15-2.72$ $(\mathrm{m}, 3 \mathrm{H}), 2.61-2.27(\mathrm{~m}, 7 \mathrm{H}), 2.25-2.04(\mathrm{~m}, 4 \mathrm{H}), 2.04-1.72(\mathrm{~m}, 8 \mathrm{H}), 1.75-1.58(\mathrm{~m}, 3 \mathrm{H}), 1.43-1.22$ $(\mathrm{m}, 8 \mathrm{H}), 1.19-0.93(\mathrm{~m}, 1 \mathrm{H}), 0.63-0.44(\mathrm{~m}, 1 \mathrm{H}) .{ }^{31} \mathrm{P}-\mathrm{NMR}\left(122 \mathrm{MHz}, \mathrm{CD}_{2} \mathrm{Cl}_{2}\right) \delta(\mathrm{ppm})=121.7 .{ }^{19} \mathrm{~F}-$ NMR $\left(282 \mathrm{MHz}, \mathrm{CD}_{2} \mathrm{Cl}_{2}\right) \delta(\mathrm{ppm})=-62.9,-63.40$. HR-MS (ESI) $\mathrm{m} / \mathrm{z}$ calcd for $\mathrm{C}_{36} \mathrm{H}_{47} \mathrm{NOF}_{3} \mathrm{PIr}[\mathrm{M}]^{+}$ 790.2977 found 790.2990 .

2.1.4.11. (+)-Iridium(I)-[[(1,2,5,6-n)-1,5-cyclooctadiene]-[7-((S)(di-cyclohexylphosphinite-kP)-2-((4-fluoro)phenyl)-4-methyl-6,7-dihydro$5 H$-cyclopenta [b]pyridine-kN]-tetrakis(3,5bis(trifluoromethyl)phenyl)borate(-) (5k)

The reaction was performed once in our laboratory using

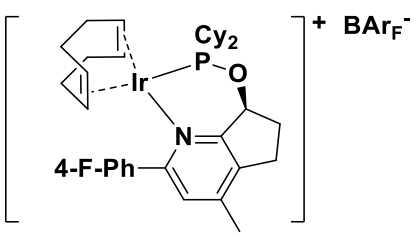

$\left[\operatorname{Ir}(\mathrm{COD})_{2}\right]_{\mathrm{BArF}_{4}}(287 \mathrm{mg}, 0.225 \mathrm{mmol})$ according to the above described procedure (1.1.4.), using $\mathbf{4 f a s ~ s t a r t i n g ~ m a t e r i a l . ~ T h e ~ c o m p l e x ~}$ could be isolated as an orange solid ( $245 \mathrm{mg} ; 70 \%$ based on $\left.\left[\operatorname{lr}(\mathrm{COD})_{2}\right] \mathrm{BAr}_{4}\right)$.

${ }^{1} \mathrm{H}-\mathrm{NMR}\left(300 \mathrm{MHz}, \mathrm{CDCl}_{3}\right): \delta(\mathrm{ppm})=8.38-8.12(\mathrm{~m}, 2 \mathrm{H}), 7.82-7.63(\mathrm{~m}, 8 \mathrm{H}), 7.51(\mathrm{~s}, 5 \mathrm{H}), 7.44-$ $7.17(\mathrm{~m}, 2 \mathrm{H}), 5.81-5.63(\mathrm{~m}, 1 \mathrm{H}), 4.81-4.67(\mathrm{~m}, 1 \mathrm{H}), 4.67-4.49(\mathrm{~m}, 1 \mathrm{H}), 3.57-3.35(\mathrm{~m}, 1 \mathrm{H}), 3.05$ $-2.90(\mathrm{~m}, 1 \mathrm{H}), 2.88-2.61(\mathrm{~m}, 3 \mathrm{H}), 2.36(\mathrm{~s}, 3 \mathrm{H}), 2.31-2.04(\mathrm{~m}, 7 \mathrm{H}), 2.01-1.73(\mathrm{~m}, 7 \mathrm{H}), 1.70-1.48$ $(\mathrm{m}, 6 \mathrm{H}), 1.42-1.20(\mathrm{~m}, 6 \mathrm{H}), 1.16-0.97(\mathrm{~m}, 4 \mathrm{H}), 0.63-0.40(\mathrm{~m}, 1 \mathrm{H}) .{ }^{31} \mathrm{P}-\mathrm{NMR}\left(122 \mathrm{MHz}, \mathrm{CDCl}_{3}\right) \delta$ $(\mathrm{ppm})=121.3 .{ }^{19} \mathrm{~F}-\mathrm{NMR}\left(282 \mathrm{MHz}, \mathrm{CDCl}_{3}\right) \delta(\mathrm{ppm})=-62.4$, -106.6. HR-MS (ESI) $\mathrm{m} / \mathrm{z}$ calcd for $\mathrm{C}_{35} \mathrm{H}_{47}$ NOFPIr [M] $]^{+} 740.3009$ found 740.3013 . 
SI: Application of Crabtree/Pfaltz-type iridium complexes for the catalyzed asymmetric hydrogenation of an agrochemical building block

2.1.4.12. (+)-Iridium(I)-[[(1,2,5,6-n)-1,5-cyclooctadiene]-[7-((R)(di-cyclohexylphosphinite-kP)-2-(anthracen-9-yl)-6,7-dihydro-5Hcyclopenta[b]pyridine-kN]-tetrakis(3,5bis(trifluoromethyl)phenyl)borate(-) (5l)

The reaction was performed once in our laboratory using

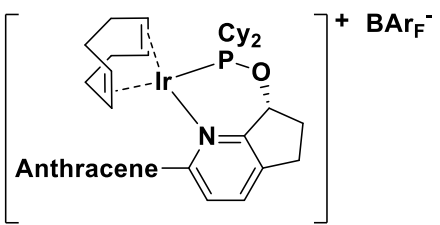
$\left[\operatorname{Ir}(\mathrm{COD})_{2}\right] \mathrm{BAr}_{4}{ }_{4}(287 \mathrm{mg}, 0.225 \mathrm{mmol})$ according to the above described procedure (1.1.4.), using $\mathbf{4} \mathbf{d}$ as starting material. The complex could be isolated after two time purification as an orange solid (151 mg; 36\% based on $\left.\left[\operatorname{Ir}(\mathrm{COD})_{2}\right] \mathrm{BAr}_{4}\right)$.

${ }^{1} \mathrm{H}-\mathrm{NMR}\left(300 \mathrm{MHz}, \mathrm{CD}_{2} \mathrm{Cl}_{2}\right): \delta(\mathrm{ppm})=8.84(\mathrm{~s}, 1 \mathrm{H}), 8.38-8.27(\mathrm{~m}, 1 \mathrm{H}), 8.21$ (ddt, $J=8.5,1.3,0.7 \mathrm{~Hz}$, $1 \mathrm{H}), 8.18-8.02(\mathrm{~m}, 2 \mathrm{H}), 7.83-7.72(\mathrm{~m}, 10 \mathrm{H}), 7.72-7.54(\mathrm{~m}, 6 \mathrm{H}), 7.49$ (ddd, $J=8.8,6.6,1.4 \mathrm{~Hz}, 1 \mathrm{H})$, $7.23-6.96(\mathrm{~m}, 1 \mathrm{H}), 5.74-5.54(\mathrm{~m}, 1 \mathrm{H}), 5.26-5.12(\mathrm{~m}, 1 \mathrm{H}), 4.41-4.18(\mathrm{~m}, 1 \mathrm{H}), 3.53-3.15(\mathrm{~m}, 3 \mathrm{H})$, $2.75-2.61(\mathrm{~m}, 2 \mathrm{H}), 2.59-2.32(\mathrm{~m}, 2 \mathrm{H}), 2.18-1.91(\mathrm{~m}, 6 \mathrm{H}), 1.92-1.74(\mathrm{~m}, 5 \mathrm{H}), 1.74-1.56(\mathrm{~m}, 2 \mathrm{H})$, $1.48-1.21(\mathrm{~m}, 10 \mathrm{H}), 1.18-0.99(\mathrm{~m}, 1 \mathrm{H}), 0.96-0.59(\mathrm{~m}, 2 \mathrm{H}), 0.39-0.15(\mathrm{~m}, 1 \mathrm{H}), 0.06-0.11(\mathrm{~m}$, 1H). ${ }^{31}$ P-NMR $\left(122 \mathrm{MHz}, \mathrm{CD}_{2} \mathrm{Cl}_{2}\right) \delta(\mathrm{ppm})=120.3 .{ }^{19} \mathrm{~F}-\mathrm{NMR}\left(282 \mathrm{MHz}, \mathrm{CD}_{2} \mathrm{Cl}_{2}\right) \delta(\mathrm{ppm})=-62.9 . \mathbf{H R}-$ MS (ESI) $\mathrm{m} / z$ calcd for $\mathrm{C}_{42} \mathrm{H}_{50} \mathrm{NOPIr}[\mathrm{M}]^{+} 808.3259$ found 808.3278 .

2.1.4.13. (+)-Iridium(I)-[[(1,2,5,6-n)-1,5-cyclooctadiene]-[7-((S)(di-cyclopentylphosphinite-kP)-4-methyl-2-(naphthalene-1-yl)-6,7dihydro-5H-cyclopenta[b]pyridine-kN]-tetrakis(3,5bis(trifluoromethyl)phenyl)borate(-) (5n)

The reaction was performed once in our laboratory using

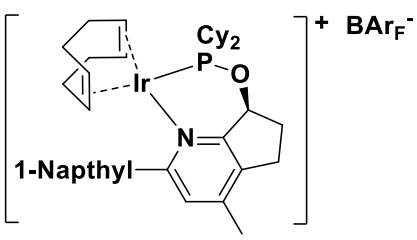
$\left[\operatorname{Ir}(\mathrm{COD})_{2}\right]_{\mathrm{BAr}_{4}}(287 \mathrm{mg}, 0.225 \mathrm{mmol})$ according to the above described procedure (1.1.4.), using $\mathbf{4} \mathbf{k}$ as starting material. The complex could be isolated as an orange solid (286 mg; 78\% based on $\left.\left[\operatorname{Ir}(\mathrm{COD})_{2}\right] \mathrm{BAr}_{4}{ }_{4}\right)$.

${ }^{1} \mathrm{H}-\mathrm{NMR}\left(300 \mathrm{MHz}, \mathrm{CD}_{2} \mathrm{Cl}_{2}\right): \delta(\mathrm{ppm})=8.61-8.48(\mathrm{~m}, 1 \mathrm{H}), 8.28-8.15(\mathrm{~m}, 1 \mathrm{H}), 8.11-7.98(\mathrm{~m}, 1 \mathrm{H})$, $7.98-7.81(\mathrm{~m}, 1 \mathrm{H}), 7.79-7.50(\mathrm{~m}, 16 \mathrm{H}), 5.70(\mathrm{ddd}, J=8.1,4.9,3.2 \mathrm{~Hz}, 1 \mathrm{H}), 5.37-5.25(\mathrm{~m}, 1 \mathrm{H}), 4.79$ (d, $J=10.4 \mathrm{~Hz}, 1 \mathrm{H}$ ), $3.53-3.41(\mathrm{~m}, 1 \mathrm{H}$ ), 3.13 (ddd, $J=17.2,9.5,4.9 \mathrm{~Hz}, 1 \mathrm{H}$ ), 2.96 (ddd, $J=17.1,9.4$, $4.9 \mathrm{~Hz}, 1 \mathrm{H}), 2.88-2.66(\mathrm{~m}, 1 \mathrm{H}), 2.49-2.34(\mathrm{~m}, 7 \mathrm{H}), 2.27-2.14(\mathrm{~m}, 1 \mathrm{H}), 2.09-1.56(\mathrm{~m}, 15 \mathrm{H}), 1.43$ $-1.12(\mathrm{~m}, 9 \mathrm{H}), 1.06-0.92(\mathrm{~m}, 1 \mathrm{H}), 0.78-0.59(\mathrm{~m}, 1 \mathrm{H}), 0.42-0.25(\mathrm{~m}, 1 \mathrm{H}) .{ }^{31}$ P-NMR $(122 \mathrm{MHz}$, $\left.\mathrm{CD}_{2} \mathrm{Cl}_{2}\right) . \delta=121.7 .{ }^{19} \mathrm{~F}-\mathrm{NMR}\left(282 \mathrm{MHz}, \mathrm{CD}_{2} \mathrm{Cl}_{2}\right) \delta=-62.9$. HR-MS (ESI) $\mathrm{m} / \mathrm{z}$ calcd for $\mathrm{C}_{39} \mathrm{H}_{50} \mathrm{NOPIr}[\mathrm{M}]^{+}$ 722.3259 found 722.3262 . 
SI: Application of Crabtree/Pfaltz-type iridium complexes for the catalyzed asymmetric hydrogenation of an agrochemical building block

2.1.4.14. (+)-Iridium(I)-[[(1,2,5,6-n)-1,5-cyclooctadiene]-[7-((S)(di-cyclopentylphosphinite-kP)-2-(4-(tert-butyl)phenyl)-4-methyl-6,7dihydro-5H-cyclopenta[b]pyridine-kN]-tetrakis(3,5bis(trifluoromethyl)phenyl)borate(-) (5o)

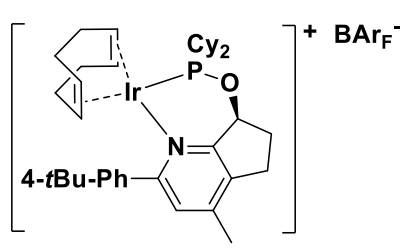

The reaction was performed once in our laboratory according to the above described procedure (1.1.4.), using $4 \mathbf{i}$ as starting material. The theoretical yield of the ligand was $>99 \%$. The complex could be isolated as an orange solid $\left(286 \mathrm{mg} ; 64 \%\right.$ based on $\left.\left[\operatorname{Ir}(\mathrm{COD})_{2}\right] \mathrm{BAr}_{4}{ }_{4}\right)$.

${ }^{1} \mathrm{H}-\mathrm{NMR}\left(300 \mathrm{MHz}, \mathrm{CDCl}_{3}\right): \delta(\mathrm{ppm})=8.20(\mathrm{~d}, \mathrm{~J}=8.2 \mathrm{~Hz}, 2 \mathrm{H}), 7.77-7.69(\mathrm{~m}, 8 \mathrm{H}), 7.66(\mathrm{~d}, \mathrm{~J}=8.4 \mathrm{~Hz}$, $2 \mathrm{H}), 7.53(\mathrm{~d}, \mathrm{~J}=4.9 \mathrm{~Hz}, 5 \mathrm{H}), 5.77-5.67(\mathrm{~m}, 1 \mathrm{H}), 4.78(\mathrm{~d}, \mathrm{~J}=7.6 \mathrm{~Hz}, 1 \mathrm{H}), 4.57(\mathrm{~s}, 1 \mathrm{H}), 3.47(\mathrm{~s}, 1 \mathrm{H})$, $3.08-2.89(\mathrm{~m}, 1 \mathrm{H}), 2.89-2.66(\mathrm{~m}, 2 \mathrm{H}), 2.59(\mathrm{p}, \mathrm{J}=7.4 \mathrm{~Hz}, 1 \mathrm{H}), 2.47-1.74(\mathrm{~m}, 15 \mathrm{H}), 1.42(\mathrm{~s}, 17 \mathrm{H})$, $1.18-0.78(\mathrm{~m}, 5 \mathrm{H}), 0.72-0.48(\mathrm{~m}, 1 \mathrm{H}) .{ }^{31} \mathbf{P}-\mathrm{NMR}\left(122 \mathrm{MHz}, \mathrm{CDCl}_{3}\right) 121.3 .{ }^{19} \mathrm{~F}-\mathrm{NMR}(282 \mathrm{MHz}, \mathrm{CDCl})$ $\delta=-62.4$. HR-MS (ESI): $m / z$ calculated for $\left[\mathrm{C}_{39} \mathrm{H}_{56} \mathrm{NOP}^{193} \mid \mathrm{r}\right]^{+}: 778.3729$ found 778.3732 .

2.1.4.15. (+)-Iridium(I)-[[(1,2,5,6-n)-1,5-cyclooctadiene]-[7-((S)(di-cyclopentylphosphinite-kP)-2-(3,5-dimethylphenyl)-4-methyl-6,7dihydro-5H-cyclopenta[b]pyridine-kN]-tetrakis(3,5bis(trifluoromethyl)phenyl)borate(-) (5p)

The reaction was performed once in our laboratory using

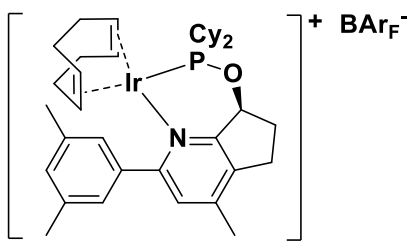
$\left[\operatorname{Ir}(\mathrm{COD})_{2}\right]_{\mathrm{BAr}_{4}}(287 \mathrm{mg}, 0.225 \mathrm{mmol})$ according to the above described procedure (1.1.4.), using 4 las starting material. The complex could be isolated using DCM (100\%) to afford an orange solid (310 mg; $85 \%$ based on $\left.\left[\operatorname{Ir}(\mathrm{COD})_{2}\right] \mathrm{BAr}_{4}\right)$.

${ }^{1} \mathrm{H}-\mathrm{NMR}\left(300 \mathrm{MHz}, \mathrm{CD}_{2} \mathrm{Cl}_{2}\right): \delta(\mathrm{ppm})=7.86(\mathrm{~s}, 2 \mathrm{H}), 7.79-7.47(\mathrm{~m}, 13 \mathrm{H}), 7.36(\mathrm{~s}, 1 \mathrm{H}), 5.79-5.62(\mathrm{~m}$, $1 \mathrm{H}), 4.78-4.74(\mathrm{~m}, 1 \mathrm{H}), 4.57-4.53(\mathrm{~m}, 1 \mathrm{H}), 3.56-3.48(\mathrm{~m}, 1 \mathrm{H}), 3.13-2.95(\mathrm{~m}, 1 \mathrm{H}), 2.95-2.61$ $(\mathrm{m}, 3 \mathrm{H}), 2.51(\mathrm{~s}, 6 \mathrm{H}), 2.47-2.36(\mathrm{~m}, 5 \mathrm{H}), 2.34-2.03(\mathrm{~m}, 5 \mathrm{H}), 2.03-1.77(\mathrm{~m}, 7 \mathrm{H}), 1.71-1.47(\mathrm{~m}$, 7H), $1.45-1.19(\mathrm{~m}, 5 \mathrm{H}), 1.19-0.98(\mathrm{~m}, 4 \mathrm{H}), 0.70-0.62(\mathrm{~m}, 1 \mathrm{H}) .{ }^{31} \mathrm{P}-\mathrm{NMR}\left(122 \mathrm{MHz}, \mathrm{CD}_{2} \mathrm{Cl}_{2}\right) \delta=$ 121.7. ${ }^{19} \mathrm{~F}-\mathrm{NMR}\left(282 \mathrm{MHz}, \mathrm{CD}_{2} \mathrm{Cl}_{2}\right) \delta=-62.9$. HR-MS (ESI) $\mathrm{m} / z$ calcd for $\mathrm{C}_{37} \mathrm{H}_{52} \mathrm{NOPIr}[\mathrm{M}]^{+} 750.3416$ found 750.3406 . 
SI: Application of Crabtree/Pfaltz-type iridium complexes for the catalyzed asymmetric hydrogenation of an agrochemical building block

2.1.4.16. (+)-Iridium(I)-[[(1,2,5,6- $)-1,5$-cyclooctadiene]-[7-((S)(di-cyclopentylphosphinite-kP)-2-(2,6-dimethylphenyl)-4-methyl-6,7dihydro-5H-cyclopenta[b]pyridine-kN]-tetrakis(3,5bis(trifluoromethyl)phenyl)borate(-) (5q)

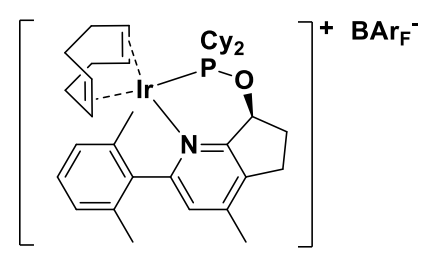

$$
\begin{aligned}
& \text { The reaction was performed once in our laboratory using } \\
& {\left[\operatorname{Ir}(\mathrm{COD})_{2}\right] \mathrm{BArF}_{4}(287 \mathrm{mg}, 0.225 \mathrm{mmol}) \text { according to the above described }} \\
& \text { procedure (1.1.4.), using } 4 \mathrm{~m} \text { as starting material. The complex could be } \\
& \text { isolated as an orange solid }\left(298 \mathrm{mg} ; 82 \% \text { based on }\left[\operatorname{Ir}(\mathrm{COD})_{2}\right] \mathrm{BAr}_{4}\right) \text {. }
\end{aligned}
$$

${ }^{1} \mathrm{H}-\mathrm{NMR}\left(300 \mathrm{MHz}, \mathrm{CD}_{2} \mathrm{Cl}_{2}\right): \delta(\mathrm{ppm})=7.80-7.52(\mathrm{~m}, 12 \mathrm{H}), 7.42-7.19(\mathrm{~m}, 3 \mathrm{H}), 7.12(\mathrm{~d}, J=7.5 \mathrm{~Hz}$, $1 \mathrm{H}), 5.65(\mathrm{td}, \mathrm{J}=5.6,2.6 \mathrm{~Hz}, 1 \mathrm{H}), 5.48-5.42(\mathrm{~m}, 1 \mathrm{H}), 4.43-4.37(\mathrm{~m}, 1 \mathrm{H}), 3.38-3.30(\mathrm{~m}, 1 \mathrm{H}), 3.21$ $-2.89(\mathrm{~m}, 3 \mathrm{H}), 2.67(\mathrm{~s}, 3 \mathrm{H}), 2.58-2.45(\mathrm{~m}, 2 \mathrm{H}), 2.42(\mathrm{~s}, 3 \mathrm{H}), 2.38-2.16(\mathrm{~m}, 2 \mathrm{H}), 2.13-2.05(\mathrm{~m}, 3 \mathrm{H})$, $2.02-1.89(\mathrm{~m}, 4 \mathrm{H}), 1.84(\mathrm{~s}, 3 \mathrm{H}), 1.81-1.72(\mathrm{~m}, 2 \mathrm{H}), 1.64-1.49(\mathrm{~m}, 3 \mathrm{H}), 1.39-1.19(\mathrm{~m}, 8 \mathrm{H}), 1.12-$ $0.99(\mathrm{~m}, 4 \mathrm{H}), 0.68-0.56(\mathrm{~m}, 1 \mathrm{H}) .{ }^{31}$ P-NMR $\left(122 \mathrm{MHz}, \mathrm{CD}_{2} \mathrm{Cl}_{2}\right) \delta=118.8 .{ }^{19} \mathrm{~F}-\mathrm{NMR}\left(282 \mathrm{MHz}, \mathrm{CD}_{2} \mathrm{Cl}_{2}\right)$ $\delta=-62.9$.HR-MS HR-MS (ESI) $\mathrm{m} / z$ calcd for $\mathrm{C}_{37} \mathrm{H}_{52} \mathrm{NOPIr}[\mathrm{M}]^{+} 750.3416$ found 750.3420 .

2.1.4.17. (+)-Iridium(I)-[[(1,2,5,6-n)-1,5-cyclooctadiene]-[7-((S)(di-cyclopentylphosphinite-kP)-2-(2,4,6-trimethylphenyl)-4-methyl-6,7dihydro-5H-cyclopenta[b]pyridine-kN]-tetrakis(3,5bis(trifluoromethyl)phenyl)borate(-) (5r) The reaction was performed once in our laboratory using

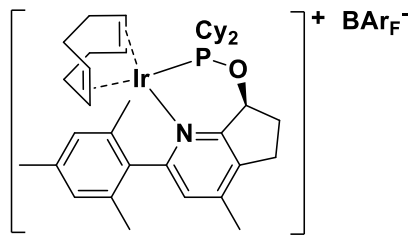
$\left[\operatorname{Ir}(\mathrm{COD})_{2}\right]_{\mathrm{BAr}_{4}}(287 \mathrm{mg}, 0.225 \mathrm{mmol})$ according to the above described procedure (1.1.4.), using $\mathbf{4 n}$ as starting material. The complex could be isolated as an orange solid (148 mg; $40 \%$ based on $\left.\left[\operatorname{lr}(\mathrm{COD})_{2}\right] \mathrm{BAr}_{4}{ }_{4}\right)$.

HR-MS (ESI) $m / z$ calcd for $\mathrm{C}_{38} \mathrm{H}_{54} \mathrm{NOPIr}[\mathrm{M}]^{+} 764.3572$ found 764.3577 .

\begin{tabular}{|c|c|c|}
\hline Cation & $\begin{array}{l}{ }^{1} \mathrm{H}-\mathrm{NMR}(400 \mathrm{MHz}, \\
\left.\mathrm{CD}_{2} \mathrm{Cl}_{2}\right): \delta(\mathrm{ppm})\end{array}$ & $\begin{array}{l}{ }^{13} \mathrm{C}-\mathrm{NMR}(1 \\
\left.\mathrm{CD}_{2} \mathrm{Cl}_{2}\right) \delta(\end{array}$ \\
\hline \multirow[t]{4}{*}{ Ligand core } & $7.21(\mathrm{~s}), 2-\mathrm{H}$ & $162.1, \mathrm{C} 8$ \\
\hline & $5.65(d t), 7-H$ & $160.6, \mathrm{C} 1$ \\
\hline & $3.10(\mathrm{~m}, 1 \mathrm{H}), 5-\mathrm{H}$ & 150.4, C3 \\
\hline & $2.96(\mathrm{ddd}, 1 \mathrm{H}), 5-\mathrm{H}$ & $138.4, \mathrm{C} 4$ \\
\hline
\end{tabular}

${ }^{11}$ B-NMR $\left(128 \mathrm{MHz}, \mathrm{CD}_{2} \mathrm{Cl}_{2}\right) \delta(\mathrm{ppm})=-6.6$.

${ }^{19} \mathrm{~F}-\mathrm{NMR}\left(377 \mathrm{MHz}, \mathrm{CD}_{2} \mathrm{Cl}_{2}\right) \delta(\mathrm{ppm})=-62.9$.

${ }^{31} \mathrm{P}\left\{{ }^{1} \mathrm{H}\right\}-\mathrm{NMR}\left(162 \mathrm{MHz}, \mathrm{CD}_{2} \mathrm{Cl}_{2}\right) \delta(\mathrm{ppm})=118.6$. 

hydrogenation of an agrochemical building block

\begin{tabular}{lll}
\hline & $2.49(\mathrm{~m}, 2 \mathrm{H}), 6-\mathrm{H}$ & $132.2, \mathrm{C} 2$ \\
& $2.41(\mathrm{~s}), 3-\mathrm{CH}_{3}$ & $86.0, \mathrm{C} 7$ \\
& & $29.7(\mathrm{~d}, 9.5 \mathrm{~Hz}), \mathrm{C} 6$ \\
& & $27.7, \mathrm{CC}$ \\
& & $19.0,3-\mathrm{CH}_{3}$ \\
\hline
\end{tabular}

$\begin{array}{lll}\text { R (mesityl) } & \\ & 6.09(\mathrm{~s}, 1 \mathrm{H}), 5-\mathrm{H} & 140.5, \mathrm{C} 4 \\ 2.64(\mathrm{~s}, 1 \mathrm{H}), 3 \mathrm{H}), \mathrm{H} & 137.9, \mathrm{CH} 2 & 137.3, \mathrm{C} 6 \\ 2.35(\mathrm{~s}, 3 \mathrm{H}), 4-\mathrm{CH}_{3} & 135.9, \mathrm{C} 1 \text { (ipso) } \\ 1.79(\mathrm{~s}, 3 \mathrm{H}), 6-\mathrm{CH}_{3} & 129.0, \mathrm{C} 3 \\ & 128.8, \mathrm{C} 5 \\ & 24.9,6-\mathrm{CH}_{3} \\ & 21.1,4-\mathrm{CH}_{3} \\ & 20.9,2-\mathrm{CH}_{3}\end{array}$

Cyclohexyl
$2.07(\mathrm{~m}), \mathrm{CH}$
$40.2(d, 29.1 \mathrm{~Hz}), \mathrm{CH}$
$1.27(\mathrm{~m}), \mathrm{CH}$
$37.0(\mathrm{~d}, 31.9 \mathrm{~Hz}), \mathrm{CH}$
$1.0-2.4(\mathrm{~m}), 5 \times \mathrm{CH}_{2}$
$24.6-28.4 ; 5 \times \mathrm{CH}_{2}$

$\begin{array}{lll}\text { Cyclooctadiene } & 5.44(\mathrm{~m}), \mathrm{CH} & 97.0(\mathrm{~d}, 8.6 \mathrm{~Hz}), \mathrm{CH} \\ 4.37(\mathrm{~m}), \mathrm{CH} & 90.9(\mathrm{~d}, 14.6 \mathrm{~Hz}), \mathrm{CH} \\ 3.33(\mathrm{~m}), \mathrm{CH} & 65.2, \mathrm{CH} \\ 3.05(\mathrm{~m}), \mathrm{CH} & 61.3, \mathrm{CH} \\ & 2.25(\mathrm{~m}, 1 \mathrm{H}), \mathrm{CH}_{2} & 38.0(\mathrm{~d}, 3.4 \mathrm{~Hz}), \mathrm{CH}_{2} \\ 2.11(\mathrm{~m}, 2 \mathrm{H}), \mathrm{CH}_{2} & 35.0(\mathrm{~d}, 1.4 \mathrm{~Hz}), \mathrm{CH}_{2} \\ 2.01(\mathrm{~m}, 1 \mathrm{H}), \mathrm{CH}_{2} & 28.1(\mathrm{~d}, 1 \mathrm{~Hz}), \mathrm{CH}_{2} \\ 1.96(\mathrm{~m}, 1 \mathrm{H}), \mathrm{CH}_{2} & 25.1(\mathrm{~d}, 2 \mathrm{~Hz}), \mathrm{CH}_{2} \\ & 1.33(\mathrm{~m}, 1 \mathrm{H}), \mathrm{CH}_{2} & \\ & 1.23(\mathrm{~m}, 1 \mathrm{H}), \mathrm{CH}_{2} & \\ & 1.04(\mathrm{~m}, 1 \mathrm{H}), \mathrm{CH}_{2} & \end{array}$


SI: Application of Crabtree/Pfaltz-type iridium complexes for the catalyzed asymmetric hydrogenation of an agrochemical building block

\begin{tabular}{|c|c|c|}
\hline Anion & $\delta\left({ }^{1} \mathrm{H}\right) / \mathrm{ppm}$ & $\delta\left({ }^{13} \mathrm{C}\right) / \mathrm{ppm}, J\left({ }^{19} \mathrm{~F},{ }^{13} \mathrm{C}\right)$ \\
\hline & $7.73(\mathrm{br}), 2-\mathrm{H} / 6-\mathrm{H}$ & $162.1\left(\mathrm{q},{ }^{1} J\left({ }^{13} \mathrm{C},{ }^{11} \mathrm{~B}\right)=49.8\right.$ \\
\hline & 7.57 (br), 4-H & Hz), C1 (ipso) \\
\hline & & $135.2(\mathrm{br}, 1 \mathrm{~Hz}), \mathrm{C} 2 / \mathrm{C} 6$ \\
\hline & & $129.2(q, 31 \mathrm{~Hz}), \mathrm{C} 3 / \mathrm{C} 5$ \\
\hline & & 124.9 (q, $272 \mathrm{~Hz}), 3-\mathrm{CF}_{3} / 5-$ \\
\hline & & $\mathrm{CF}_{3}$ \\
\hline & & $117.8(m, 4 \mathrm{~Hz}), \mathrm{C} 4$ \\
\hline
\end{tabular}

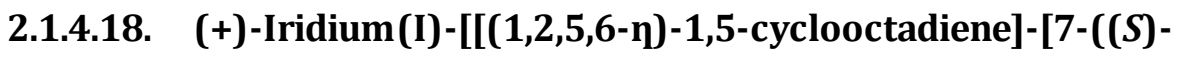

(di-cyclohexylphosphinite-kP)-2-(anthracen-9-yl)-4methyl-6,7-dihydro$5 \mathrm{H}$-cyclopenta[b]pyridine-kN]-tetrakis(3,5-

bis(trifluoromethyl)phenyl)borate(-) (5s)

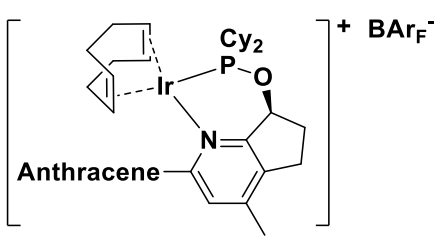

The reaction was performed once in our laboratory using $\left[\operatorname{lr}(\mathrm{COD})_{2}\right] \mathrm{BAr}_{4}(287 \mathrm{mg}, 0.225 \mathrm{mmol})$ according to the above described procedure (1.1.4.), using $\mathbf{4} \mathbf{j}$ as starting material. The complex could be isolated using DCM (100\%) to afford an orange solid (296 mg; 78\% based on $\left.\left[\operatorname{Ir}(\mathrm{COD})_{2}\right] \mathrm{BAr}_{4}\right)$.

${ }^{1} \mathrm{H}-\mathrm{NMR}\left(300 \mathrm{MHz}, \mathrm{CD}_{2} \mathrm{Cl}_{2}\right): \delta(\mathrm{ppm})=8.68(\mathrm{~s}, 1 \mathrm{H}), 8.23-7.85(\mathrm{~m}, 3 \mathrm{H}), 7.75-7.23(\mathrm{~m}, 17 \mathrm{H}), 7.05$ (dq, $J=8.8,1.0 \mathrm{~Hz}, 1 \mathrm{H}), 5.61-5.40(\mathrm{~m}, 2 \mathrm{H}), 5.12-4.88(\mathrm{~m}, 1 \mathrm{H}), 4.24-4.00(\mathrm{~m}, 1 \mathrm{H}), 3.25-2.88(\mathrm{~m}$, $3 \mathrm{H}), 2.58-2.46(\mathrm{~m}, 2 \mathrm{H}), 2.44-2.14(\mathrm{~m}, 7 \mathrm{H}), 2.08-1.61(\mathrm{~m}, 11 \mathrm{H}), 1.61-1.37(\mathrm{~m}, 5 \mathrm{H}), 1.37-1.07$ $(\mathrm{m}, 6 \mathrm{H}), 1.03-0.85(\mathrm{~m}, 1 \mathrm{H}), 0.65-0.45(\mathrm{~m}, 1 \mathrm{H}), 0.16(\mathrm{dtd}, J=15.8,10.4,5.6 \mathrm{~Hz}, 1 \mathrm{H}),-0.16(\mathrm{dt}, J=$ 13.2, $9.1 \mathrm{~Hz}, 1 \mathrm{H}) .{ }^{31} \mathrm{P}-\mathrm{NMR}\left(122 \mathrm{MHz}, \mathrm{CD}_{2} \mathrm{Cl}_{2}\right) \delta=120.6 .{ }^{19} \mathrm{~F}-\mathrm{NMR}\left(282 \mathrm{MHz}, \mathrm{CD}_{2} \mathrm{Cl}_{2}\right) \delta=-62.9 . \mathbf{H R}-$ MS (ESI) $\mathrm{m} / z$ calcd for $\mathrm{C}_{43} \mathrm{H}_{52} \mathrm{NOPIr}[\mathrm{M}]^{+} 822.3416$ found 822.3416 .

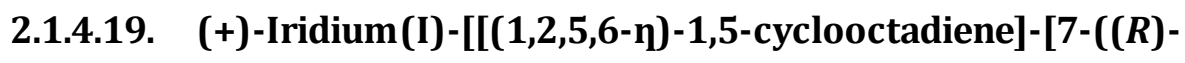
(di-cyclohexylphosphinite-kP))-2-methyl-6,7-dihydro-5Hcyclopenta $[b]$ pyridine- $\mathrm{kN}]$-tetrakis $(3,5-$ bis(trifluoromethyl)phenyl)borate $(-)(5 \mathrm{~m})$

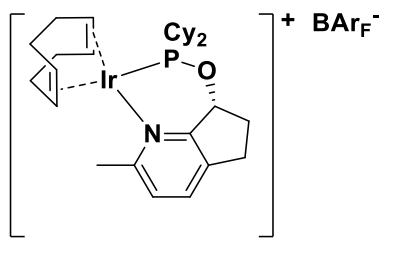

The reaction was performed once in our laboratory using $\left[\operatorname{Ir}(\mathrm{COD})_{2}\right]_{\mathrm{BAr}_{4}}(287 \mathrm{mg}, 0.225 \mathrm{mmol})$ according to the above described procedure (1.1.4.), using $\mathbf{4 0}$ as starting material. The complex could be isolated using DCM (100\%) as an orange solid (249 $\mathrm{mg} ; 73 \%$ based on $\left[\mathrm{Ir}(\mathrm{COD})_{2}\right] \mathrm{BAr}_{4}{ }_{4}$. 
SI: Application of Crabtree/Pfaltz-type iridium complexes for the catalyzed asymmetric hydrogenation of an agrochemical building block

${ }^{1} \mathrm{H}-\mathrm{NMR}\left(300 \mathrm{MHz}, \mathrm{CD}_{2} \mathrm{Cl}_{2}\right): \delta(\mathrm{ppm})=7.81-7.61(\mathrm{~m}, 9 \mathrm{H}), 7.56(\mathrm{~d}, J=2.0 \mathrm{~Hz}, 4 \mathrm{H}), 7.34(\mathrm{~d}, J=8.0$ $\mathrm{Hz}, 1 \mathrm{H}), 5.76(\mathrm{dt}, J=8.7,4.5 \mathrm{~Hz}, 1 \mathrm{H}), 5.05-4.84(\mathrm{~m}, 2 \mathrm{H}), 3.74-3.57(\mathrm{~m}, 1 \mathrm{H}), 3.56-3.36(\mathrm{~m}, 1 \mathrm{H})$, $3.07(\mathrm{~s}, 3 \mathrm{H}), 3.01-1.49(\mathrm{~m}, 23 \mathrm{H}), 1.42-1.01(\mathrm{~m}, 9 \mathrm{H}), 0.85-0.70(\mathrm{~m}, 1 \mathrm{H}), 0.51-0.25(\mathrm{~m}, 1 \mathrm{H})$. 31P-NMR $\left(122 \mathrm{MHz}, \mathrm{CD}_{2} \mathrm{Cl}_{2}\right) \delta(\mathrm{ppm})=126.2 .{ }^{19} \mathrm{~F}-\mathrm{NMR}\left(282 \mathrm{MHz}, \mathrm{CD}_{2} \mathrm{Cl}_{2}\right) \delta(\mathrm{ppm})=-62.9$. HR-MS (ESI) $\mathrm{m} / z$ calcd for $\mathrm{C}_{29} \mathrm{H}_{44} \mathrm{NOPIr}[\mathrm{M}]^{+} 644.2766$ found 644.2762 .

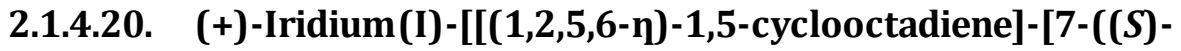
(di-cyclohexylphosphinite-kP))-(3,5-di-tert-butyl-4-methoxyphenyl)-4methyl-6,7-dihydro-5H-cyclopenta[b]pyridine-kN]-tetrakis(3,5bis(trifluoromethyl)phenyl)borate(-) (5t)

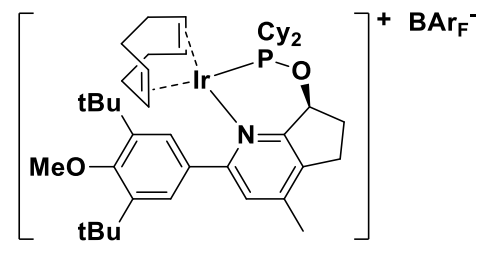

The reaction was performed once in our laboratory using $\left[\operatorname{Ir}(\mathrm{COD})_{2}\right]_{\mathrm{BAr}_{4}}(287 \mathrm{mg}, 0.225 \mathrm{mmol})$ according to the above described procedure (1.1.4.), using $4 p$ as starting material. The complex could be isolated using DCM (100\%) as an orange solid (164 $\mathrm{mg} ; 42 \%$ based on $\left.\left[\operatorname{Ir}(\mathrm{COD})_{2}\right] \mathrm{BAr}_{4}{ }_{4}\right)$.

${ }^{1} \mathrm{H}-\mathrm{NMR}\left(300 \mathrm{MHz}, \mathrm{CD}_{2} \mathrm{Cl}_{2}\right): \delta(\mathrm{ppm})=7.86-7.62(\mathrm{~m}, 10 \mathrm{H}), 7.56(\mathrm{~s}, 4 \mathrm{H}), 7.38(\mathrm{~s}, 1 \mathrm{H}), 5.72(\mathrm{dt}, J=$ 8.1, $5.2 \mathrm{~Hz}, 1 \mathrm{H}), 4.85-4.63(\mathrm{~m}, 2 \mathrm{H}), 3.80(\mathrm{~s}, 3 \mathrm{H}), 3.49-3.30(\mathrm{~m}, 1 \mathrm{H}), 3.18-2.60(\mathrm{~m}, 4 \mathrm{H}), 2.54-$ $2.23(\mathrm{~m}, 6 \mathrm{H}), 2.23-1.57(\mathrm{~m}, 16 \mathrm{H}), 1.53-1.49(\mathrm{~m}, 20 \mathrm{H}), 1.46-0.93(\mathrm{~m}, 10 \mathrm{H}) .{ }^{31} \mathbf{P}-\mathbf{N M R}(122 \mathrm{MHz}$, $\left.\mathrm{CD}_{2} \mathrm{Cl}_{2}\right) \delta(\mathrm{ppm})=123.3 .{ }^{19} \mathrm{~F}-\mathrm{NMR}\left(282 \mathrm{MHz}, \mathrm{CD}_{2} \mathrm{Cl}_{2}\right) \delta(\mathrm{ppm})=-62.9$. HR-MS (ESI) $\mathrm{m} / \mathrm{z}$ calcd for $\mathrm{C}_{44} \mathrm{H}_{66} \mathrm{NO}_{2} \mathrm{PIr}[\mathrm{M}]^{+} 864.4460$ found 864.4448 .

2.1.4.21. (+)-Iridium(I)-[[(1,2,5,6- $)-1,5-c y c l o o c t a d i e n e]-[7-((S)-$ (di-cyclohexylphosphinite-kP))-(2,4,6-tri-iso-propylphenyl)-4-methyl6,7-dihydro-5H-cyclopenta $[b]$ pyridine- $\mathrm{kN}]$-tetrakis $(3,5-$ bis(trifluoromethyl)phenyl)borate(-) (5u)

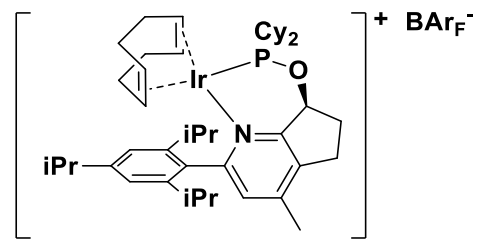

The reaction was performed once in our laboratory using [Ir(COD) $)_{2}$ BArF $_{4}(287 \mathrm{mg}, 0.225 \mathrm{mmol})$ according to the above described procedure (1.1.4.), using $\mathbf{4 q}$ as starting material. After three times purification using Heptane/DCM (1/1), the complex could be isolated as an orange solid $(51 \mathrm{mg} ; 14 \%$ based on $\left.\left[\operatorname{Ir}(\mathrm{COD})_{2}\right] \mathrm{BAr}_{4}\right)$.

${ }^{1} \mathrm{H}-\mathrm{NMR}\left(400 \mathrm{MHz}, \mathrm{CD}_{2} \mathrm{Cl}_{2}\right): \delta(\mathrm{ppm})=7.80-7.64(\mathrm{~m}, 8 \mathrm{H}), 7.56(\mathrm{~s}, 4 \mathrm{H}), 7.23(\mathrm{~s}, 2 \mathrm{H}), 7.04(\mathrm{~s}, 1 \mathrm{H})$, $5.65(\mathrm{dt}, J=5.9,3.7 \mathrm{~Hz}, 1 \mathrm{H}), 5.45-5.35(\mathrm{~m}, 1 \mathrm{H}), 4.04(\mathrm{ddd}, J=8.2,5.4,3.6 \mathrm{~Hz}, 1 \mathrm{H}), 3.34(\mathrm{dd}, J=$ $11.2,6.4 \mathrm{~Hz}, 1 \mathrm{H}), 3.19-3.08(\mathrm{~m}, 3 \mathrm{H}), 3.06-2.89(\mathrm{~m}, 2 \mathrm{H}), 2.56-2.44(\mathrm{~m}, 2 \mathrm{H}), 2.41(\mathrm{~s}, 3 \mathrm{H}), 2.33-$ $1.84(\mathrm{~m}, 9 \mathrm{H}), 1.84-1.43(\mathrm{~m}, 15 \mathrm{H}), 1.35-1.24(\mathrm{~m}, 12 \mathrm{H}), 1.23-1.14(\mathrm{~m}, 5 \mathrm{H}), 1.09$ (dd, $J=10.0,6.8$ 
SI: Application of Crabtree/Pfaltz-type iridium complexes for the catalyzed asymmetric hydrogenation of an agrochemical building block

$\mathrm{Hz}, 6 \mathrm{H}), 0.95(\mathrm{~d}, J=6.6 \mathrm{~Hz}, 3 \mathrm{H}), 0.60-0.46(\mathrm{~m}, 1 \mathrm{H}) .{ }^{31}$ P-NMR $\left(162 \mathrm{MHz}, \mathrm{CD}_{2} \mathrm{Cl}_{2}\right) \delta(\mathrm{ppm})=119.4$.

${ }^{19} \mathrm{~F}-\mathrm{NMR}\left(282 \mathrm{MHz}, \mathrm{CD}_{2} \mathrm{Cl}_{2}\right) \delta(\mathrm{ppm})=-62.9$. HR-MS $(\mathrm{ESI}) \mathrm{m} / \mathrm{z}$ calcd for $\mathrm{C}_{44} \mathrm{H}_{66} \mathrm{NOPIr}[\mathrm{M}]^{+} 848.4511$ found 848.4512 .

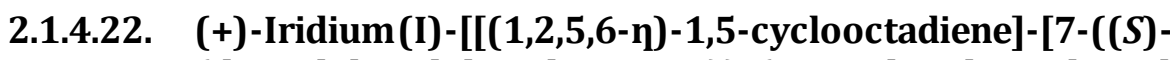

(di-cyclohexylphosphinite-kP))-(4-tert-butyl-2,6-dimethylylphenyl)-4methyl-6,7-dihydro-5H-cyclopenta[b]pyridine-kN]-tetrakis(3,5bis(trifluoromethyl)phenyl)borate(-) (5v)

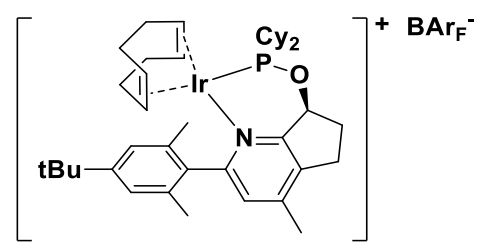

The reaction was performed once in our laboratory using $\left[\operatorname{Ir}(\mathrm{COD})_{2}\right]_{\mathrm{BAr}^{\mathrm{F}}}(287 \mathrm{mg}, 0.225 \mathrm{mmol})$ according to the above described procedure (1.1.4.), using $4 r$ as starting material. The complex could be isolated as an orange solid ( $274 \mathrm{mg} ; 73 \%$ based on $\left.\left[\operatorname{Ir}(\mathrm{COD})_{2}\right] \mathrm{BAr}_{4}\right)$ using Heptane/DCM (1/1).

${ }^{1} \mathrm{H}-\mathrm{NMR}\left(300 \mathrm{MHz}, \mathrm{CD}_{2} \mathrm{Cl}_{2}\right): \delta(\mathrm{ppm})=7.79-7.66(\mathrm{~m}, 8 \mathrm{H}), 7.56(\mathrm{~s}, 4 \mathrm{H}), 7.29(\mathrm{~s}, 1 \mathrm{H}), 7.23(\mathrm{~s}, 1 \mathrm{H})$, $7.13(\mathrm{~s}, 1 \mathrm{H}), 5.65(\mathrm{td}, \mathrm{J}=5.9,2.2 \mathrm{~Hz}, 1 \mathrm{H}), 5.46-5.40(\mathrm{~m}, 1 \mathrm{H}), 4.42-4.36(\mathrm{~m}, 1 \mathrm{H}), 3.38-3.30$ $(\mathrm{m}, 1 \mathrm{H}), 3.19-2.86(\mathrm{~m}, 3 \mathrm{H}), 2.65(\mathrm{~s}, 3 \mathrm{H}), 2.59-2.44(\mathrm{~m}, 2 \mathrm{H}), 2.42(\mathrm{~s}, 3 \mathrm{H}), 2.38-1.54(\mathrm{~m}, 2 \mathrm{H})$, $1.46-0.98(\mathrm{~m}, 21 \mathrm{H}), 0.70-0.58(\mathrm{~m}, 1 \mathrm{H}) .{ }^{31} \mathrm{P}-\mathrm{NMR}\left(122 \mathrm{MHz}, \mathrm{CD}_{2} \mathrm{Cl}_{2}\right) \delta(\mathrm{ppm})=118.7 .{ }^{19} \mathrm{~F}-\mathrm{NMR}$ $\left(282 \mathrm{MHz}, \mathrm{CD}_{2} \mathrm{Cl}_{2}\right) \delta(\mathrm{ppm})=-62.9$. HR-MS $(\mathrm{ESI}) \mathrm{m} / \mathrm{z}$ calcd for $\mathrm{C}_{41} \mathrm{H}_{60} \mathrm{NOPIr}[\mathrm{M}]^{+} 806.4042$ found 806.4053 . 


\section{SI: Application of Crabtree/Pfaltz-type iridium complexes for the catalyzed asymmetric hydrogenation of an agrochemical building block}

\section{Results for the Hydrogenation of 1}

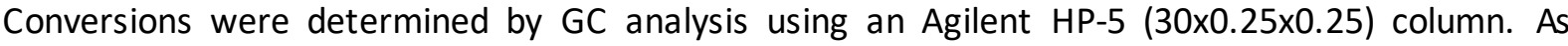
temperature program 50/8-120/0/15-200/0/25-300/10 was used. The carrier gas is He and the flow is $1.0 \mathrm{~mL} / \mathrm{min}$.

The enantiomeric excess was determined by HPLC analysis using a Reposil column (from Altmann Analytik). When a ratio of heptane to ethanol of 95 to 5 and a flow of $1.0 \mathrm{~mL} / \mathrm{min}$ was used the retention times were as following: Starting material $4.750 \mathrm{~min},(R)$-enantiomer $5.312 \mathrm{~min}$ and $(S)$-enantiomer $6.247 \mathrm{~min}$. When a ratio of heptane to ethanol of 99 to 1 and a flow of $0.5 \mathrm{~mL} / \mathrm{min}$ was used the retention times were as following: Starting material $18.041 \mathrm{~min},(R)$-enantiomer $23.194 \mathrm{~min}$ and $(S)$-enantiomer $30.844 \mathrm{~min}$. An alternative separation method is as following: Phenomex Lux $5 \mu \mathrm{m}$ Amylose-2 LC column (150x4.6 mm), heptane/ethanol: 90 to 10; flow $1.0 \mathrm{~mL} / \mathrm{min}$. Retention times: $3.087 \mathrm{~min}(R)$ and $6.798(S)$.

\subsection{Setup}

- $300 \mathrm{~mL}$ Parr autoclave (up to 200 bar)

- External temperature detection

- Internal stirring (“Tic Tac" stirring bar)

- up to seven experiment parallel

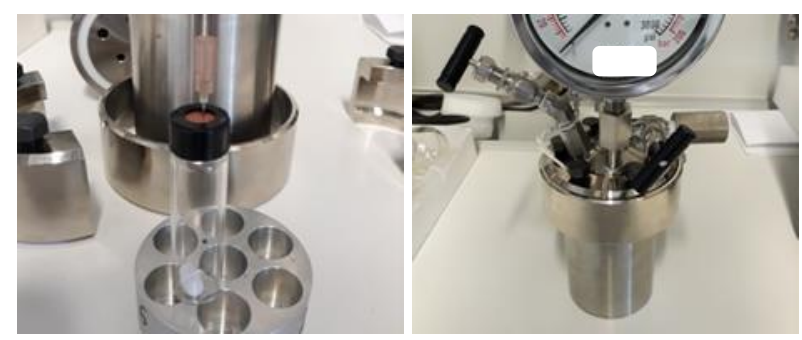

\subsection{Hydrogenation using new complexes}

General experimental procedure: The Ir-complex (catalyst loading given) and the substrate $(3 \mathrm{mmol})$ were placed in an 8-mL autoclave vial containing a PTFE-coated stirring bar. The autoclave vial was closed using a screw cap with septum, placed in a fitted metal plate, connected to a schlenk line and subsequently flushed with argon (10 min). HFIP ( $4 \mathrm{~mL}$ ) was added via the septum to the vial. The vial was placed in an argon containing autoclave and the autoclave was flushed with argon (10 min). The autoclave was pressurized with hydrogen gas (10 bar) and subsequently depressurized to atmospheric pressure three times. After this the autoclave was pressurized to 60 bar hydrogen pressure and was placed in a suitable alumina block. After heating to $85^{\circ} \mathrm{C}\left(\sim 4^{\circ} \mathrm{C} / \mathrm{min} ; 15 \mathrm{~min}\right)$ the reaction was kept at this temperature for the given time. The autoclave was placed in an ice bath to allow fast cooling to room temperature. After depressurizing, the vial was taken out of the autoclave and the reactions outcome was determined by GC-FID analysis (diluted with EtOH) and the enantiomeric excess by HPLC analysis. 


\section{SI: Application of Crabtree/Pfaltz-type iridium complexes for the catalyzed asymmetric hydrogenation of an agrochemical building block}

\section{(R)-1-(2,2,4-trimethyl-3,4-dihydroquinolin-1(2H)-yl)ethan-1-one (2)}

Chemical Formular: $\mathrm{C}_{14} \mathrm{H}_{19} \mathrm{NO}$<smiles>CC(=O)N1c2ccccc2C(C)CC1(C)C</smiles>

Molecular Weight: $217.15 \mathrm{~g} / \mathrm{mol}$

The product was isolated as an yellowish oil by flash column chromatography using heptane:EtOAc with a gradient starting from 100:0 going to 50:50. The product elutes at a ratio of heptane to ethyl acetate of $60: 40$.

${ }^{1} \mathrm{H}-\mathrm{NMR}\left(300 \mathrm{MHz}, \mathrm{CD}_{2} \mathrm{Cl}_{2}\right): \delta(\mathrm{ppm})=7.23-7.09(\mathrm{~m}, 3 \mathrm{H}), 6.98-6.90(\mathrm{~m}, 1 \mathrm{H}), 2.85-2.69(\mathrm{~m}$, $1 \mathrm{H}), 2.03(\mathrm{~s}, 3 \mathrm{H}), 1.83(\mathrm{dd}, \mathrm{J}=12.8,2.6 \mathrm{~Hz}, 1 \mathrm{H}), 1.67(\mathrm{~s}, 3 \mathrm{H}), 1.46(\mathrm{~s}, 3 \mathrm{H}), 1.32(\mathrm{~d}, \mathrm{~J}=6.8 \mathrm{~Hz}, 3 \mathrm{H})$, $1.24-1.13(\mathrm{~m}, 1 \mathrm{H})$.

${ }^{13} \mathrm{C}-\mathrm{NMR}\left(75 \mathrm{MHz}, \mathrm{CD}_{2} \mathrm{Cl}_{2}\right) \delta(\mathrm{ppm})=171.9,141.50,140.0,126.2,125.9,125.1,123.2,59.2,52.9$, $29.6,28.0,26.3,26.1,17.2$.

MS: (El, $70 \mathrm{eV}) \mathrm{m} / \mathrm{z}=217$ ([M] $\left.]^{+}, 20\right), 161$ (14), 160 (100), 144 (14).

\subsection{Deuteration of 1 using $5 r$}

Complex $5 \mathrm{r}(0.025 \mathrm{~mol} \%)$ and $\mathbf{1}(3 \mathrm{mmol})$ were placed in an 8- $\mathrm{mL}$ autoclave vial containing a PTFEcoated stirring bar. The autoclave vial was closed using a screw cap with septum, placed in a fitted metal plate, connected to a Schlenk line and subsequently flushed with argon (10 min). HFIP (4 $\mathrm{mL}$ ) was added via the septum to the vial. The vial was placed in an argon containing autoclave and the autoclave was flushed with argon (10 $\mathrm{min})$. The autoclave was pressurized with deuterium gas (10 bar) and subsequently depressurized to atmospheric pressure three times. After this the autoclave was pressurized (due to experimental limitations a pressure of only 40 bar instead of the usual 60 bar was applied) and was placed in a suitable alumina block. After heating to $85^{\circ} \mathrm{C}\left(\sim 4^{\circ} \mathrm{C} / \mathrm{min} ; 15 \mathrm{~min}\right)$ the reaction was kept at this temperature for the given time. The autoclave was placed in an ice bath to allow fast cooling to room temperature. After depressurizing, the vial was taken out of the autoclave. All volatiles were removed under reduced pressure and the crude product was subsequently purified by column chromatography on silica using ethyl acetate/heptanes $(1: 1, v / v)$ as the eluent.

${ }^{1} \mathrm{H}-\mathrm{NMR}\left(400 \mathrm{MHz}, \mathrm{CDCl}_{3}\right): \delta(\mathrm{ppm})=7.20-7.10(\mathrm{~m}, 3 \mathrm{H}), 6.95-6.90(\mathrm{~m}, 1 \mathrm{H}), 2.07(\mathrm{~s}, 3 \mathrm{H}), 1,83$ (s, $0.07(\mathrm{C} 1) \mathrm{H}), 1.70(\mathrm{~s}, 3 \mathrm{H}), 1.48(\mathrm{~s}, 3 \mathrm{H}), 1.31(\mathrm{~s}, 2,64(\mathrm{C} 2) \mathrm{H}), 1.24-1.13(\mathrm{~m}, 1(\mathrm{C} 3) \mathrm{H})$. 
SI: Application of Crabtree/Pfaltz-type iridium complexes for the catalyzed asymmetric hydrogenation of an agrochemical building block

${ }^{13} \mathrm{C}-\mathrm{NMR}(75 \mathrm{MHz}, \mathrm{CDCl}) \delta(\mathrm{ppm})=172.0,163.5,140.2,138.3,126.5,125.5,124.3,122.9,120.4$, 59.0, $51.9(\mathrm{t}, J=15 \mathrm{~Hz}), 28.7(\mathrm{t}, J=15 \mathrm{~Hz}), 27.8,26.1,26.0,16.9$.

HR-MS (ESI) $m / z$ calcd for $\mathrm{C}_{14} \mathrm{H}_{18} \mathrm{D}_{2} \mathrm{NO}[\mathrm{M}+\mathrm{H}]^{+} 220.1667$ found 220.1670.
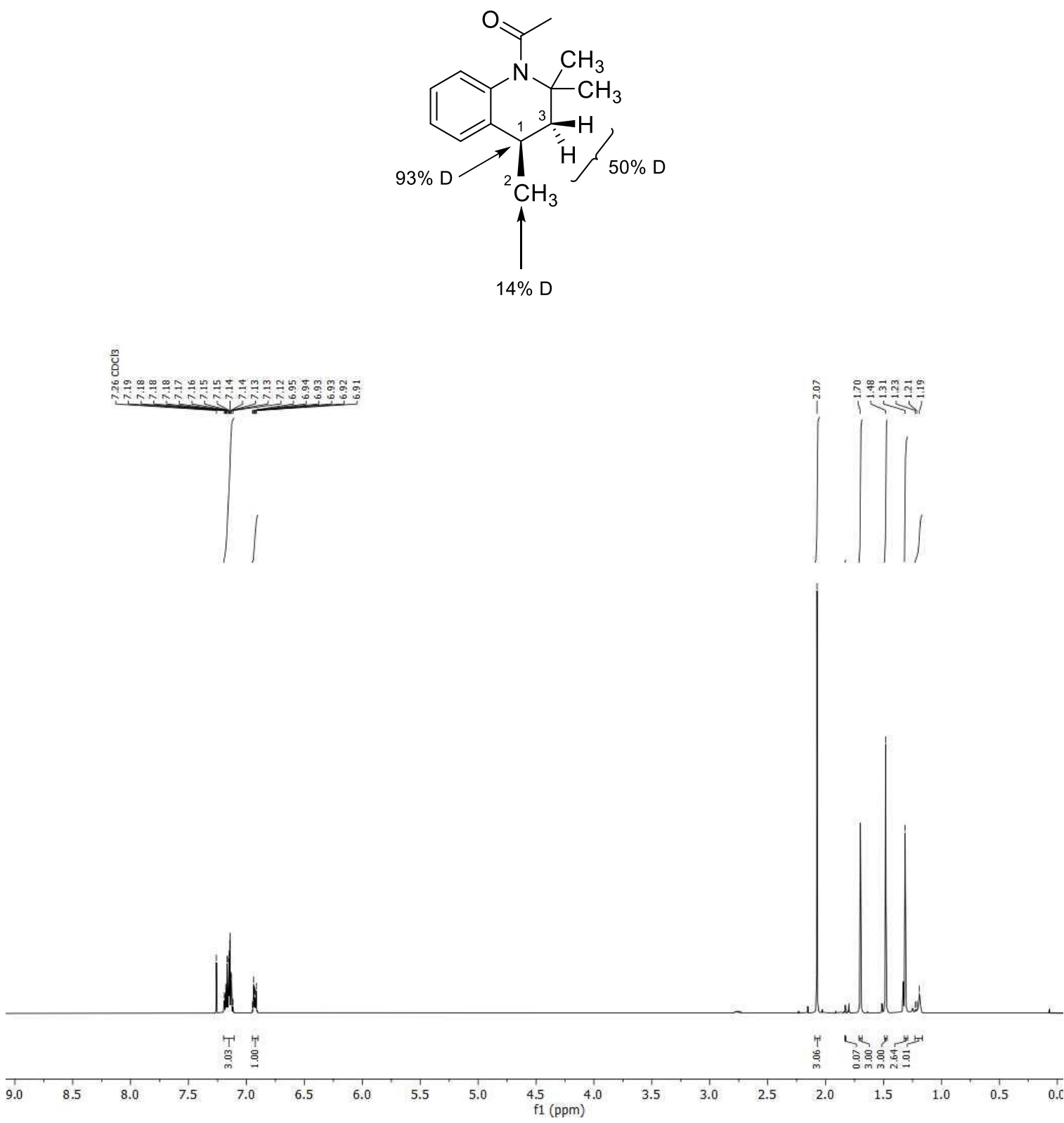

${ }^{1} \mathrm{H}-\mathrm{NMR}$ of 2 in $\mathrm{CDCl}_{3}$ after deuteration. 
SI: Application of Crabtree/Pfaltz-type iridium complexes for the catalyzed asymmetric hydrogenation of an agrochemical building block
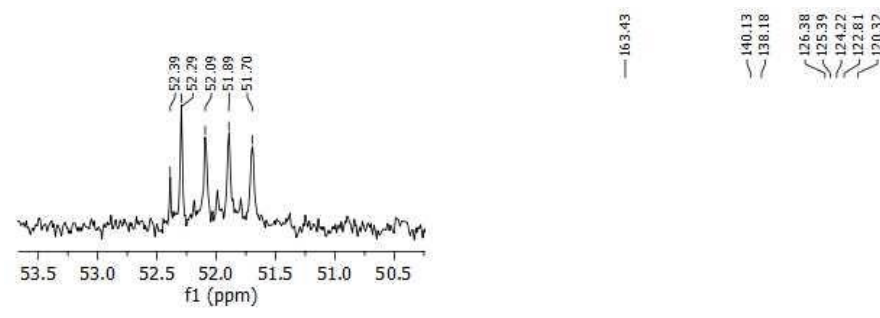

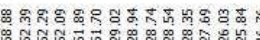

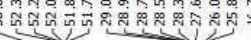
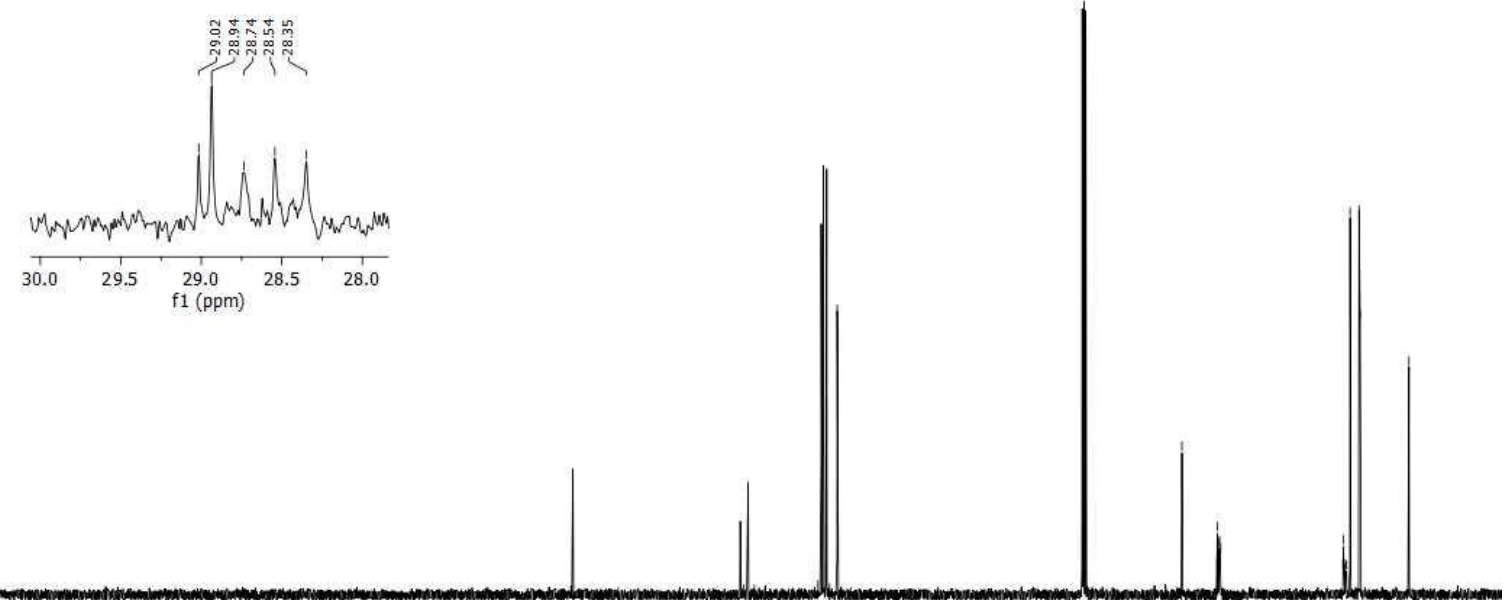

$\begin{array}{llllllllllllllllllllllllllllllllllllllll}270 & 260 & 250 & 240 & 230 & 220 & 210 & 200 & 190 & 180 & 170 & 160 & 150 & 140 & 130 & 120 & 110 & 100 & 90 & 80 & 70 & 60 & 50 & 40 & 30 & 20 & 10 & 0\end{array}$

${ }^{13} \mathrm{C}-\mathrm{NMR}$ of 2 in $\mathrm{CDCl}_{3}$ after deuteration

4. Other catalysts tested for the asymmetric hydrogenation of 1

$$
\text { Entry }
$$

Catalyst

Conversion $(\% \mathrm{a} / \mathrm{a}$

ee

HPLC)

(\%)

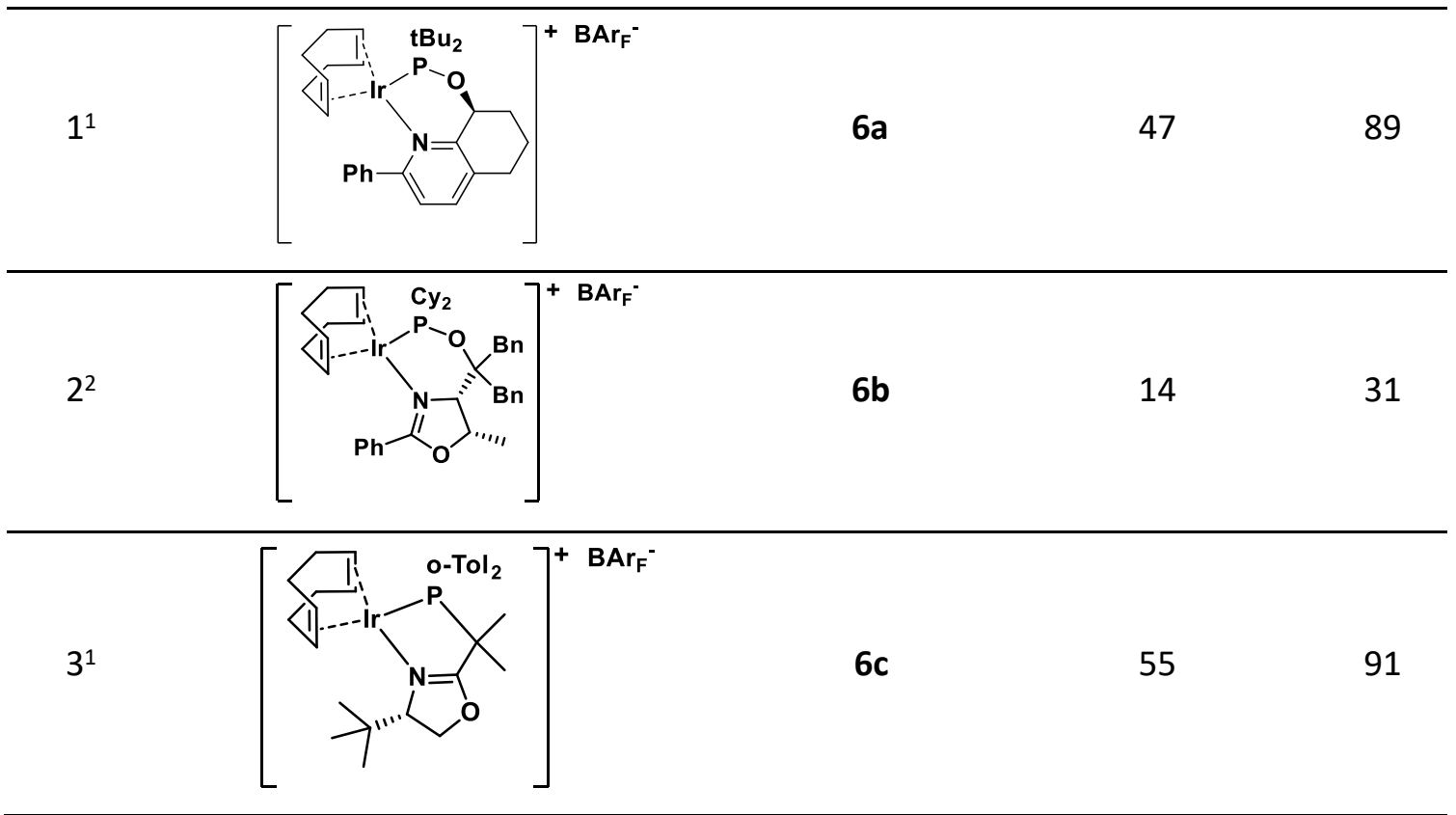


SI: Application of Crabtree/Pfaltz-type iridium complexes for the catalyzed asymmetric hydrogenation of an agrochemical building block

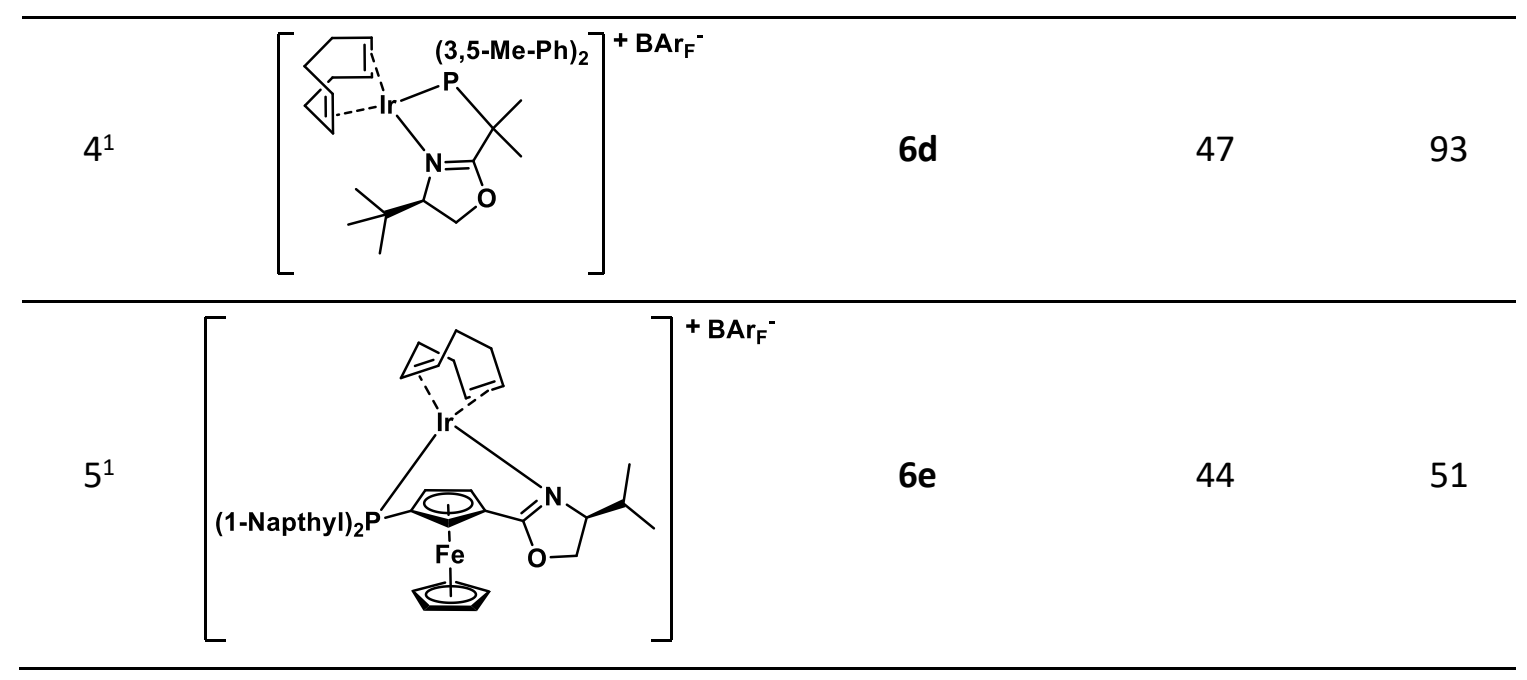

${ }^{1}$ For reference see: WO2019/185541 A1.

${ }^{2}$ For reference see: DE112015001290T5.

\section{Optimization of reaction parameters}

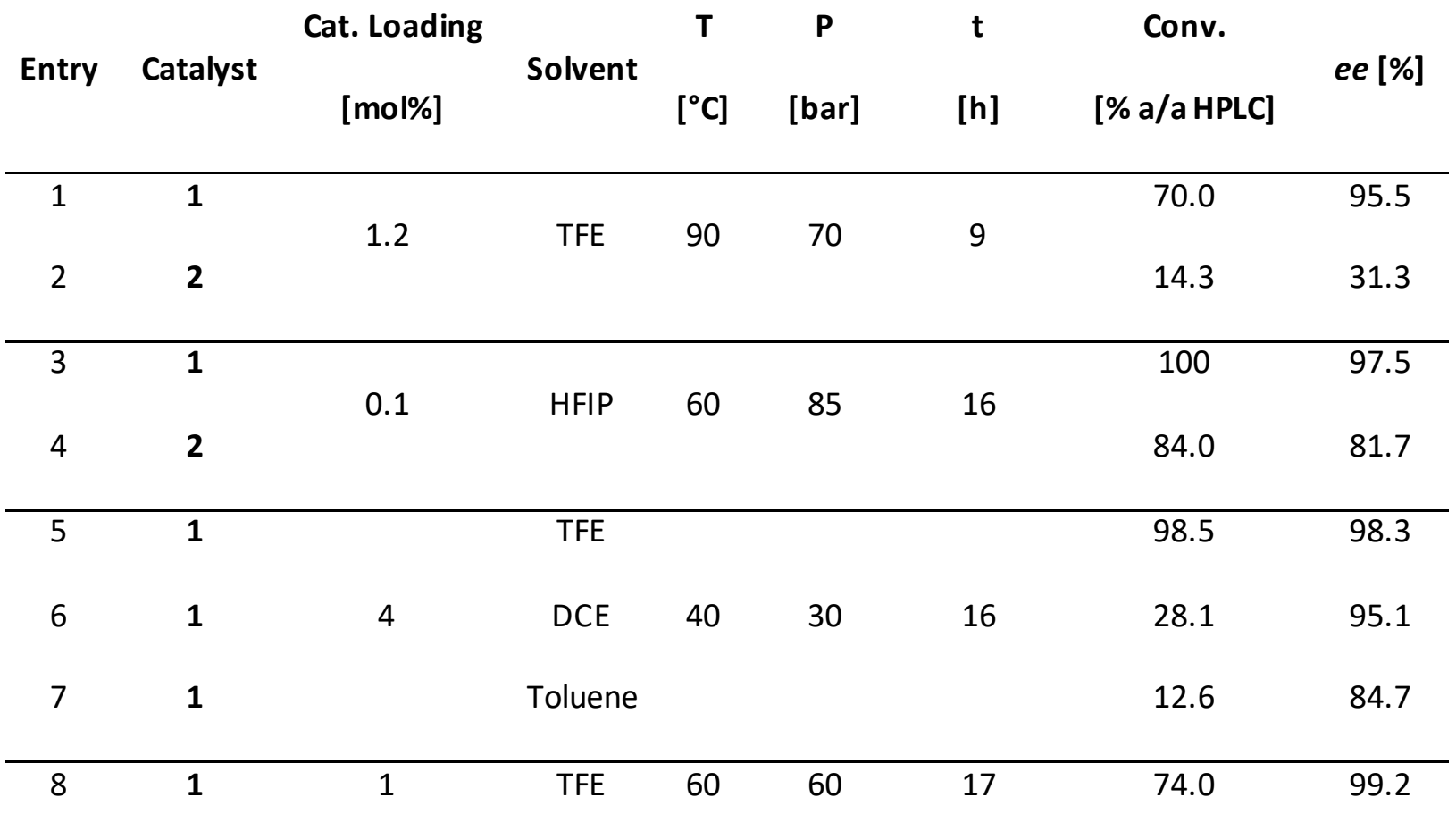

